\title{
DECOMPOSITION NUMBERS FOR WEIGHT THREE BLOCKS OF SYMMETRIC GROUPS AND IWAHORI-HECKE ALGEBRAS
}

\author{
MATTHEW FAYERS
}

\begin{abstract}
Let $\mathbb{F}$ be a field, $q$ a non-zero element of $\mathbb{F}$ and $\mathcal{H}_{n}=\mathcal{H}_{\mathbb{F}, q}\left(\mathfrak{S}_{n}\right)$ the Iwahori-Hecke algebra of the symmetric group $\mathfrak{S}_{n}$. If $B$ is a block of $\mathcal{H}_{n}$ of $e$-weight 3 and the characteristic of $\mathbb{F}$ is at least 5 , we prove that the decomposition numbers for $B$ are all at most 1 . In particular, the decomposition numbers for a $p$-block of $\mathfrak{S}_{n}$ of defect 3 are all at most 1 .
\end{abstract}

\section{INTRODUCTION}

Let $\mathbb{F}$ be a field of any characteristic; we adopt the convention that a field whose prime subfield is infinite has infinite characteristic. Let $q$ be a non-zero element of $\mathbb{F}$ and let $n$ be a non-negative integer. In this paper, we discuss the decomposition numbers for the Iwahori-Hecke algebra $\mathcal{H}_{n}=\mathcal{H}_{\mathbb{F}, q}\left(\mathfrak{S}_{n}\right)$ of the symmetric group $\mathfrak{S}_{n}$. In the special case where $q=1$, this algebra is simply the group algebra $\mathbb{F} \mathfrak{S}_{n}$. We let $e$ be the least positive integer such that $1+q+\cdots+q^{e-1}=0$ in $\mathbb{F}$ if such an integer exists, and let $e=\infty$ otherwise. Each block of $\mathcal{H}_{n}$ has an $e$-weight, and in this paper we examine the blocks whose $e$-weight is 3 . The main result of the paper is as follows.

Theorem 1.1. Suppose $\operatorname{char}(\mathbb{F}) \geq 5$, and that $B$ is a block of $\mathcal{H}_{\mathbb{F}, q}\left(\mathfrak{S}_{n}\right)$ of e-weight 3. Let $\lambda$ and $\mu$ be partitions in $B$ with $\mu$ e-regular. Then $\left[S_{B}^{\lambda}: D_{B}^{\mu}\right] \leq 1$.

This result has been conjectured for some time and has proved elusive until now. In the special case of symmetric group algebras, Martin and Russell [13] have published a purported proof of this result; however, various errors have subsequently been found in that paper. In particular, when $e=p=5, \lambda=\left(8^{2}, 4,1\right)$ and $\mu=(12,9)$, the decomposition number $\left[S^{\lambda}: D^{\mu}\right]$ is hard to calculate; it was eventually found to be 1 rather than 2 by a large computer calculation carried out by Lübeck and Müller. The novelty in the present paper is to prove Theorem 1.1 first in the case where $\mathbb{F}$ has infinite characteristic, using a reverse induction with the class of 'Rouquier blocks' as a base case. We then complete the proof by showing that the 'adjustment matrices' for weight 3 blocks are trivial, verifying James's Conjecture for weight 3 blocks.

Note that if all the decomposition numbers for a block are known to be at most 1 , then these decomposition numbers can all be calculated using the Jantzen-Schaper

Received by the editors April 12, 2004 and, in revised form, July 28, 2005 and September 29, 2005

2000 Mathematics Subject Classification. Primary 20C30, $20 \mathrm{C} 08$.

An earlier version of this paper was written while the author was a research fellow at Magdalene College, Cambridge. 
formula (Theorem 1.6 below). Thus in principle we now know the decomposition numbers for weight 3 blocks of Iwahori-Hecke algebras. However, we do not have anything like a combinatorial description of these, as we do for blocks of weight 2 .

We now indicate the layout of the paper. After summarising all the background theory and notation that we shall need in the remainder of this introduction, we list some essential properties of weight 3 blocks in Section 2, mostly following Martin and Russell. We then proceed with the proof of Theorem 1.1. In Section 3, we prove Theorem 1.1 in the case where $\mathbb{F}$ has infinite characteristic; in this case, the Iwahori-Hecke algebra is better understood, and we have at our disposal a key theorem due to James and Mathas, which says that the decomposition matrices in infinite characteristic are 'independent of $e$ ', in a sense which we make precise below. In Section 4, we use the result of Section 3 to complete the proof of Theorem 1.1 , by finding the 'adjustment matrices' for weight 3 blocks.

1.1. Background and notation. Excellent references for the representation theory of the symmetric groups and the Iwahori-Hecke algebras are the books of James [6] and Mathas [14], respectively. We take most of our notation from these books, but the Specht modules we use are those defined by Dipper and James in [3] rather than those in [14]. From now on we denote by $\mathcal{H}_{n}$ the Iwahori-Hecke algebra $\mathcal{H}_{\mathbb{F}, q}\left(\mathfrak{S}_{n}\right)$, and we assume that $e=\inf \left\{d \in \mathbb{N} \mid 1+q+\cdots+q^{d-1}=0\right\}$ is finite. For each partition $\lambda$ of $n$, one defines a Specht module $S_{\mathbb{F}, q}^{\lambda}$ for $\mathcal{H}_{n}$. If $\lambda$ is $e$-regular (i.e. if it does not have $e$ equal positive parts), then $S_{\mathbb{F}, q}^{\lambda}$ has an irreducible cosocle $D_{\mathbb{F}, q}^{\lambda}$; the modules $D_{\mathbb{F}, q}^{\lambda}$ give a complete set of irreducible modules for $\mathcal{H}_{n}$ as $\lambda$ ranges over the set of $e$-regular partitions of $n$. We may write $S_{\mathbb{F}, q}^{\lambda}$ and $D_{\mathbb{F}, q}^{\lambda}$ as $S_{B}^{\lambda}$ and $D_{B}^{\lambda}$ to indicate that they lie in a block $B$ of $\mathcal{H}_{n}$, or simply as $S^{\lambda}$ and $D^{\lambda}$ if $\mathbb{F}$ and $q$ are understood.

Given partitions $\lambda$ and $\mu$ of $n$ with $\mu$ e-regular, we define the decomposition number $d_{\lambda \mu}$ to be the composition multiplicity $\left[S^{\lambda}: D^{\mu}\right]$; the decomposition matrix for $\mathcal{H}_{n}$ is a matrix with rows indexed by partitions of $n$ and columns by $e$-regular partitions of $n$, in which the $(\lambda, \mu)$ entry is $d_{\lambda \mu}$. In the case $q=1$, this is the decomposition matrix in the usual representation-theoretic sense.

We use some notational conventions for modules. We write

$$
M \sim M_{1}^{a}+\cdots+M_{r}^{a}
$$

to indicate that $M$ has a filtration in which the factors are $M_{1}, \ldots, M_{r}$, each appearing $a$ times. We also write $M^{\oplus r}$ to indicate the direct sum of $r$ isomorphic copies of $M$.

We assume throughout the paper that the reader is familiar with the combinatorics of Young diagrams, particularly removable nodes and rim hooks.

1.1.1. Blocks and the abacus. If $e$ is finite, then partitions of $n$ are conveniently represented on an abacus. If $\lambda$ is a partition, choose an integer $r$ greater than the number of parts of $\lambda$, and define

$$
\beta_{i}=\lambda_{i}+r-i
$$

for $i=1, \ldots, r$. The set $\left\{\beta_{1}, \ldots, \beta_{r}\right\}$ is then said to be a set of beta-numbers for $\lambda$. Now take an abacus with $e$ vertical runners $1, \ldots, e$ from left to right, and number the positions on runner $i$ as $i-1, i-1+e, i-1+2 e, \ldots$ from the top downwards (so each non-negative integer occurs on exactly one runner). Then place a bead on 
the abacus at position $\beta_{i}$ for each $i$. The resulting configuration is said to be an abacus display for $\lambda$. The partition whose abacus display is obtained from this by moving all the beads as far up their runners as they will go is called the e-core of $\lambda$; it is a partition of $n$-we for some $w$, which is called the $e$-weight (or simply the weight) of $\lambda$. Moving a bead up $s$ spaces on its runner corresponds to removing a rim hook of length es from the Young diagram. 'Nakayama's Conjecture' [14, Corollary 5.38] says that two Specht modules $S^{\lambda}$ and $S^{\mu}$ lie in the same block of $\mathcal{H}_{n}$ (we shall abuse notation by saying that $\lambda$ and $\mu$ lie in this block) if and only if they have the same $e$-core; this means that they also have the same weight, and this is called the $(e-)$ weight of the block. If there is a bead in an abacus display for $\lambda$ with exactly $w$ empty spaces above it on the same runner, we say that the bead has weight $w$.

We shall often be comparing the numbers of beads on runners $i-1$ and $i$, and moving beads 'one space to the right' or 'one space to the left'. We wish to include the possibility $i=1$ here, with the convention that the position 'one space to the left' of position $e x$ on runner 1 is position $e x-1$ on runner $e$. To say that 'there are $\kappa$ more beads on runner $i$ than on runner $i-1$ ' in the case $i=1$ will actually mean that there are $\kappa+1$ more beads on runner 1 than runner $e$. 'Swapping runners $i-1$ and $i$ ' in the case $i=1$ will actually mean moving each bead at a position $e x>0$ on runner 1 to position $e x-1$ on runner $e$, and vice versa.

1.1.2. Branching rules. There is a natural embedding $\mathcal{H}_{n-1} \leq \mathcal{H}_{n}$. If $M$ is a module for $\mathcal{H}_{n}$, we write $M \downarrow_{\mathcal{H}_{n-r}}$ to indicate the restriction of $M$ to $\mathcal{H}_{n-r}$, and $M \uparrow \mathcal{H}_{n+r}$ to indicate the module obtained by inducing $M$ to $\mathcal{H}_{n+r}$. If $B$ is a block of $\mathcal{H}_{n-r}$ or $\mathcal{H}_{n+r}$, we write $M \downarrow_{B}$ (respectively, $M \uparrow^{B}$ ) to indicate the projection of $M \downarrow_{\mathcal{H}_{n-r}}$ (respectively, $M \uparrow^{\mathcal{H}_{n+r}}$ ) onto $B$. In this section we describe the induction and restriction of Specht modules and simple modules.

Suppose $A, B$ and $C$ are blocks of $\mathcal{H}_{n-\kappa}, \mathcal{H}_{n}$ and $\mathcal{H}_{n+\kappa}$, respectively, and that there is an abacus display for $B$ and an integer $i$ such that an abacus display for $A$ is obtained by moving $\kappa$ beads from runner $i$ to runner $i-1$, while an abacus display for $C$ is obtained by moving $\kappa$ beads from runner $i-1$ to runner $i$.

Suppose $\lambda$ is a partition in $B$, and let $\lambda^{-1}, \ldots, \lambda^{-r}$ be the partitions in $A$ which may be obtained from $\lambda$ by moving exactly $\kappa$ beads on runner $i$ one place to the left. Similarly, let $\lambda^{+1}, \ldots, \lambda^{+s}$ be the partitions in $C$ which may be obtained from $\lambda$ by moving exactly $\kappa$ beads from runner $i-1$ one place to the right. Then we have the following.

Theorem 1.2 (The Branching Rule [14, Corollary 6.2]). Suppose A, B, C and $\lambda$ are as above. Then

$$
S^{\lambda} \downarrow_{A}^{B} \sim\left(S^{\lambda^{-1}}\right)^{\kappa !}+\cdots+\left(S^{\lambda^{-r}}\right)^{\kappa !}
$$

and

$$
S^{\lambda} \uparrow_{B}^{C} \sim\left(S^{\lambda^{+1}}\right)^{\kappa !}+\cdots+\left(S^{\lambda^{+s}}\right)^{\kappa !} .
$$

The induction and restriction of simple modules is rather more subtle. Suppose $A, B, C$ and $\lambda$ are as above, and that $\lambda$ is $e$-regular. The $i$-signature of $\lambda$ is a sequence of signs defined as follows. Starting from the top row of the abacus and working down, write a - if there is a bead on runner $i$ but no bead on runner $i-1$ in the same row; write a + if there is a bead on runner $i-1$ but no bead on runner $i$ in the same row; otherwise, write nothing for that row. 
Given the $i$-signature of $\lambda$, successively delete all neighbouring pairs of the form -+ ; the resulting sequence is called the reduced $i$-signature of $\lambda$. If there are any - signs in the reduced $i$-signature, then we say that the corresponding beads on runner $i$ are normal; if there are at least $\kappa$ normal beads, then we define $\lambda^{-}$to be the partition obtained by moving the $\kappa$ highest normal beads one place to the left. If there are any + signs in the reduced $i$-signature, then we say that the corresponding beads on runner $i-1$ of the abacus display are conormal. If there are at least $\kappa$ conormal beads, then we define $\lambda^{+}$to be the partition obtained by moving the $\kappa$ lowest conormal beads one place to the right.

Theorem 1.3 ([1, $§ 2.5])$. Suppose $A, B, C$ and $\lambda$ are as above.

- If there are fewer than $\kappa$ normal beads on runner $i$ of the abacus for $\lambda$, then $D^{\lambda} \downarrow_{A}^{B}=0$.

- If there are exactly $\kappa$ normal beads on runner $i$ of the abacus for $\lambda$, then $D^{\lambda} \downarrow_{A}^{B} \cong\left(D^{\lambda^{-}}\right)^{\oplus \kappa !}$.

- If there are fewer than $\kappa$ conormal beads on runner $i-1$ of the abacus for $\lambda$, then $D^{\lambda} \uparrow_{B}^{C}=0$.

- If there are exactly $\kappa$ conormal beads on runner $i-1$ of the abacus for $\lambda$, then $D^{\lambda} \uparrow_{B}^{C} \cong\left(D^{\lambda^{-}}\right) \oplus \kappa$ !

Example. Take $e=3, i=2$ and $\kappa=1$ and suppose that

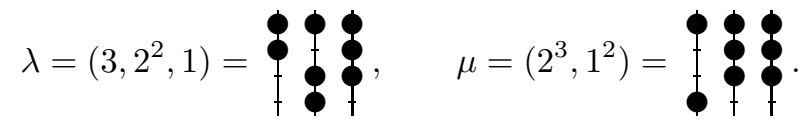

Then

$$
D^{\lambda} \uparrow_{B}^{C}=D^{\lambda^{+}}, \quad D^{\mu} \downarrow_{A}^{B} \cong D^{\mu^{-}}, \quad D^{\mu} \uparrow_{B}^{C}=0,
$$

where

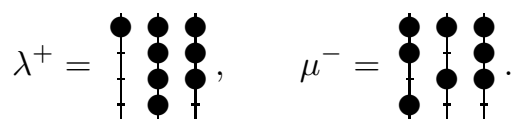

1.1.3. The Mullineux map. Let $T_{1}, \ldots, T_{n-1}$ be the standard generators of $\mathcal{H}_{n}$. Let $\sharp: \mathcal{H}_{n} \rightarrow \mathcal{H}_{n}$ be the involutory automorphism sending $T_{i}$ to $q-1-T_{i}$, and let * : $\mathcal{H}_{n} \rightarrow \mathcal{H}_{n}$ be the anti-automorphism sending $T_{i}$ to $T_{i}$. Given a module $M$ for $\mathcal{H}_{n}$, define $M^{\sharp}$ to be the module with the same underlying vector space and with the action

$$
h \cdot m=h^{\sharp} m,
$$

and define $M^{*}$ to be the module with underlying vector space dual to $M$ and with $\mathcal{H}_{n}$-action

$$
h \cdot f(m)=f\left(h^{*} m\right) .
$$

If $M$ lies in a block $B$, then $M^{\sharp}$ lies in a block $B^{\sharp}$, which we call the conjugate block to $B$.

(Note that in the symmetric group case $q=1, M^{\sharp}$ is simply $M \otimes \operatorname{sgn}$, where sgn is the 1-dimensional signature representation, while $M^{*}$ is the usual dual module to $M$.)

The effect of these functors on Specht modules is easily described; let $\lambda^{\prime}$ denote the partition conjugate to $\lambda$.

Lemma 1.4 ([14, Exercise 3.14(iii)]). For any partition $\lambda$,

$$
S^{\lambda^{\prime}} \cong\left(S^{\lambda}\right)^{\sharp *} \text {. }
$$


Now we turn to the simple modules $D^{\lambda}$, for $\lambda e$-regular. It follows from the cellularity of $\mathcal{H}_{n}$ that $\left(D^{\lambda}\right)^{*} \cong D^{\lambda}$. If we let $\lambda^{\diamond}$ denote the $e$-regular partition such that $\left(D^{\lambda}\right)^{\sharp} \cong D^{\lambda^{\diamond}}$, then $\diamond$ is an involutory bijection from the set of $e$-regular partitions of $n$ to itself. This bijection is given combinatorially by Mullineux's algorithm [15]; we shall not describe this here, but we note that given an $e$-regular partition $\lambda$, the partition $\lambda^{\diamond}$ depends only on $\lambda$ and $e$, not on the underlying field.

Of course, the functor $M \mapsto M^{\sharp}$ is a self-equivalence of the category of $\mathcal{H}_{n}$ modules, and we have the following consequence for decomposition numbers.

Corollary 1.5. For any partitions $\lambda$ and $\mu$ with $\mu$ e-regular,

$$
\left[S^{\lambda}: D^{\mu}\right]=\left[S^{\lambda^{\prime}}: D^{\mu^{\diamond}}\right]
$$

1.1.4. The Jantzen-Schaper formula. One of the most important tools in finding the decomposition numbers for $\mathcal{H}_{n}$ is the ( $q$-analogue of the) Jantzen-Schaper formula. We describe this very briefly.

Given partitions $\lambda$ and $\mu$ of $n$ and given $e$ and $p$, let $H(\lambda, \mu)$ be the set of ordered pairs $(g, h)$, where

- $g$ is a rim hook of the Young diagram $[\lambda]$ of $\lambda$;

- $h$ is a rim hook of the Young diagram $[\mu]$ of $\mu$;

- $[\lambda] \backslash g=[\mu] \backslash h$.

Now define

$$
c_{\lambda, \mu}=\sum_{(g, h) \in H(\lambda, \mu)}(-1)^{l(g)+l(h)+1} \nu_{e, p}(|g|) ;
$$

here $|g|$ is the number of nodes of $g$ and $l(g)$ its leg length, and

$$
\nu_{e, p}(x)= \begin{cases}0 & (e \nmid x), \\ 1 & (e \mid x \text { and } p=\infty), \\ 1+\nu_{p}(x / e) & (e \mid x \text { and } p<\infty)\end{cases}
$$

for a positive integer $x$.

A weak form of the Jantzen-Schaper formula may now be stated as follows, where $\geq$ indicates the lexicographic order of partitions.

Theorem 1.6 ([9, Theorem 4.7]). Let $\mathbb{F}$ be a field of characteristic p. For partitions $\lambda \neq \mu$ of $n$ with $\mu$ e-regular, define

$$
n_{\lambda, \mu}=\sum_{\nu>\lambda} c_{\lambda, \nu}\left[S_{\mathbb{F}}^{\nu}: D_{\mathbb{F}}^{\mu}\right]
$$

Then

$$
\left[S_{\mathbb{F}}^{\lambda}: D_{\mathbb{F}}^{\mu}\right] \leq n_{\lambda, \mu},
$$

and $\left[S_{\mathbb{F}}^{\lambda}: D_{\mathbb{F}}^{\mu}\right]=0$ if and only if $n_{\lambda, \mu}=0$.

In view of Theorem 1.6, we define a 'dominance' order on the set of partitions of $n$. We define $\lambda \triangleright \mu$ if $\lambda>\mu$ and $c_{\lambda, \mu} \neq 0$, and we extend transitively. Note that this does not coincide with the usual dominance order (which is a refinement), and that it depends on $e$ and $p$. In practice, though, we shall only be considering partitions of $e$-weight less than $p$, for which the order $\unrhd$ depends only on $e$.

It is clear that $\unrhd$ is reversed by conjugation of partitions, and in view of the results of Section 1.1.3, we have the following. 
Proposition 1.7. If $\mathbb{F}$ is a field of characteristic $p$, and $\lambda$ and $\mu$ are partitions of $n$ with $\mu$ e-regular and with $\lambda \neq \mu^{\diamond \prime}$, define

$$
n_{\lambda, \mu}^{\prime}=\sum_{\nu \triangleleft \lambda} c_{\lambda, \nu}\left[S_{\mathbb{F}}^{\nu}: D_{\mathbb{F}}^{\mu}\right]
$$

Then

$$
n_{\lambda, \mu}^{\prime} \geq\left[S_{\mathbb{F}}^{\lambda}: D_{\mathbb{F}}^{\mu}\right]
$$

and $n_{\lambda, \mu}^{\prime}=0$ if and only if $\left[S_{\mathbb{F}}^{\lambda}: D_{\mathbb{F}}^{\mu}\right]=0$.

Hence $\left[S^{\lambda}: D^{\mu}\right]=0$ unless $\mu \unrhd \lambda \unrhd \mu^{\diamond \prime}$.

Proof. Replace $\lambda$ and $\mu$ with $\lambda^{\prime}$ and $\mu^{\diamond}$, and apply Theorem 1.6 (replacing $\nu>\lambda^{\prime}$ with $\nu \triangleright \lambda^{\prime}$ ) and Corollary 1.5.

1.1.5. The Scopes equivalence. Various Morita equivalences for blocks of the same weight were found by Scopes [17]; although her paper was concerned only with blocks of the symmetric group, her results are known to be valid for the IwahoriHecke algebras.

Suppose that $A$ is a block of $\mathcal{H}_{n-\kappa}$ of weight $w$, and $B$ a block of $\mathcal{H}_{n}$ of weight $w$. Suppose that there is an abacus display for $B$ and an integer $i$ such that:

- there are exactly $\kappa$ more beads on runner $i$ than on runner $i-1$;

- by interchanging runners $i$ and $i-1$, we obtain an abacus display for $A$.

Then we say that $A$ and $B$ form a $[w: \kappa]$-pair.

Suppose that $A$ and $B$ form a $[w: \kappa]$-pair with $w \leq \kappa$, and let $\lambda$ be a partition in $B$. Then there are exactly $\kappa$ beads on runner $i$ in the abacus display for $\lambda$ which do not have beads immediately to their left. If we move each of these beads one place to the left, we obtain a partition in $A$, which we denote by $\Phi(\lambda)$. Then we have the following.

Theorem 1.8 ([14, p. 127]). Let $A, B$ and $\Phi$ be as above. Then:

- $\Phi$ is a bijection between the set of partitions in $B$ and the set of partitions in $A$;

- $\Phi(\lambda)$ is e-regular if and only if $\lambda$ is e-regular;

- for any partition $\lambda$ in $B$,

$$
S^{\lambda} \downarrow_{A}^{B} \sim\left(S^{\Phi(\lambda)}\right)^{\kappa !}, \quad S^{\Phi(\lambda)} \uparrow_{A}^{B} \sim\left(S^{\lambda}\right)^{\kappa !} ;
$$

- for any e-regular partition $\lambda$ in $B$,

$$
D^{\lambda} \downarrow_{A}^{B} \cong\left(D^{\Phi(\lambda)}\right)^{\oplus \kappa !}, \quad D^{\Phi(\lambda)} \uparrow_{A}^{B} \cong\left(D^{\lambda}\right)^{\oplus \kappa !} ;
$$

- the correspondence $D^{\lambda} \leftrightarrow D^{\Phi(\lambda)}$ is induced by a Morita equivalence between $B$ and $A$.

In view of Theorem 1.8, we define blocks to be Scopes equivalent if they form a $[w: \kappa]$-pair for some $\kappa \geq w$. We extend this transitively to define an equivalence relation on the set of blocks of weight $w$, and we refer to an equivalence class as a Scopes class.

It will be useful later to use the notion of $[w: \kappa]$-pairs to define a partial order on the set of blocks of a given weight. If $A$ and $B$ form a $[w: \kappa]$-pair (not necessarily with $w \leq \kappa)$, we write $A \prec B$, and extend $\preccurlyeq$ transitively to form a partial order on the set of weight $w$ blocks.

We also define a partial order on the set of Scopes classes by setting $\mathcal{C} \preccurlyeq \mathcal{D}$ if $A \preccurlyeq B$ for some $A \in \mathcal{C}$ and $B \in \mathcal{D}$, and extending transitively. It is not immediately 
obvious that this relation is anti-symmetric, but this will follow from the section on pyramids below.

1.1.6. Pyramids. In order to understand the combinatorics of Scopes classes, Richards [16] defined the notion of a pyramid. Let $\gamma$ be an $e$-core, and choose an abacus display for $\gamma$. Let $p_{1}<\cdots<p_{e}$ be those integers such that there is a bead at position $p_{i}$ but no bead at position $p_{i}+e$, for each $i$. Then exactly one $p_{i}$ lies in each congruence class modulo $e$. We renumber the runners of the abacus so that the bead at position $p_{i}$ lies on runner $i$ for each $i$. Note that we use this new numbering for the remainder of this paper. For $i<j$ the integer $p_{j}-p_{i}$ is a positive integer not divisible by $e$, and it does not depend on the choice of abacus display for $\gamma$. Given $w \geq 0$, we define

$$
{ }_{i} a_{j}=\left\{\begin{array}{cl}
w-1 & \left(e>p_{j}-p_{i}>0\right) \\
w-2 & \left(2 e>p_{j}-p_{i}>e\right) \\
& \vdots \\
1 & \left((w-1) e>p_{j}-p_{i}>(w-2) e\right) \\
0 & \left(p_{j}-p_{i}>(w-1) e\right)
\end{array}\right.
$$

for $1 \leq i<j \leq e$. For ease of notation, we also define ${ }_{0} a_{j}={ }_{j} a_{e+1}=0$ for all $j$. If $B$ is the block of $\mathcal{H}_{n}$ with core $\gamma$ and weight $w$, then the set of integers ${ }_{i} a_{j}$ is called the pyramid for $B$; we shall write ${ }_{i} a_{j}(B)$ when it is not clear to which block we are referring. We shall also use shorthand such as ${ }_{i} 0_{j}$ to indicate that ${ }_{i} a_{j}=0$.

A critical property of pyramids is the following.

Proposition 1.9 ([16, Lemma 3.1 and Proposition 3.3]). Two blocks of weight w are Scopes equivalent if and only if they have the same pyramid.

By examining the difference between the pyramids of two blocks forming a $[w: \kappa]$ pair, we can easily see the following.

Lemma 1.10. Let $A$ and $B$ be blocks of weight $w$. If $A \preccurlyeq B$, then ${ }_{i} a_{j}(A) \geq{ }_{i} a_{j}(B)$ for all $i, j$. In particular, the relation $\preccurlyeq$ on Scopes classes is anti-symmetric.

1.1.7. The row and column removal theorems. Here we state two useful results concerning decomposition numbers for Hecke algebras.

Theorem 1.11 ([14, p. 125, Rule 8]).

(1) Suppose $\lambda$ and $\mu$ are partitions of $n$ with $\mu$ e-regular, and that $\lambda_{1}=\mu_{1}$. Define

$$
\bar{\lambda}=\left(\lambda_{2}, \lambda_{3}, \ldots\right), \quad \bar{\mu}=\left(\mu_{2}, \mu_{3}, \ldots\right) .
$$

Then $\bar{\mu}$ is e-regular, and

$$
\left[S_{\mathbb{F}}^{\lambda}: D_{\mathbb{F}}^{\mu}\right]=\left[S_{\mathbb{F}}^{\bar{\lambda}}: D_{\mathbb{F}}^{\bar{\mu}}\right]
$$

for any field $\mathbb{F}$.

(2) Suppose $\lambda$ and $\mu$ are partitions of $n$ with $\mu$ e-regular, and that $\lambda_{1}^{\prime}=\mu_{1}^{\prime}$. Define

$\bar{\lambda}=\left(\max \left(\lambda_{1}-1,0\right), \max \left(\lambda_{2}-1,0\right), \ldots\right), \quad \bar{\mu}=\left(\max \left(\mu_{1}-1,0\right), \max \left(\mu_{2}-1,0\right), \ldots\right)$. 
Then $\bar{\mu}$ is e-regular, and

$$
\left[S_{\mathbb{F}}^{\lambda}: D_{\mathbb{F}}^{\mu}\right]=\left[S_{\mathbb{F}}^{\bar{\lambda}}: D_{\mathbb{F}}^{\bar{\mu}}\right]
$$

for any field $\mathbb{F}$.

1.1.8. The runner removal theorem. Here we state a result which will be very useful in Section 3; it describes a relationship between the decomposition matrices of Iwahori-Hecke algebras defined over fields of infinite characteristic with different values of $e$.

Suppose $\mathbb{F}$ has infinite characteristic, that $e \geq 3$, that $q$ is a primitive $e$ th root of unity in $\mathbb{F}$ and that $q^{\prime}$ is a primitive $(e-1)$ th root of unity in $\mathbb{F}$. Suppose $\lambda$ and $\mu$ are partitions of $n$ and suppose $r \geq \lambda_{1}^{\prime}, \mu_{1}^{\prime}$. Consider the abacus displays for $\lambda$ and $\mu$ on an abacus with $r$ beads and $e$ runners, and suppose that there are no beads on runner $i$ in either of these abacus displays. Delete runner $i$ from both displays, and let $\lambda^{-}$and $\mu^{-}$be the partitions given by the resulting abacus displays.

Theorem 1.12 ([10, Corollary 2.3]). Let $\lambda$ and $\mu$ be as above. If $\mu^{-}$is $(e-1)$ regular and if $\left|\lambda^{-}\right|=\left|\mu^{-}\right|$, then

$$
\left[S_{\mathbb{F}, q}^{\lambda}: D_{\mathbb{F}, q}^{\mu}\right]=\left[S_{\mathbb{F}, q^{\prime}}^{\lambda^{-}}: D_{\mathbb{F}, q^{\prime}}^{\mu^{-}}\right]
$$

Remark. In practice, if we are trying to calculate the decomposition number $\left[S^{\lambda}\right.$ : $\left.D^{\mu}\right]$, then we may assume that $\lambda$ and $\mu$ lie in the same block. This automatically implies that $\left|\lambda^{-}\right|=\left|\mu^{-}\right|$.

1.1.9. Adjustment matrices. Finally we come to a result which relates the decomposition matrices of Iwahori-Hecke algebras with the same value of $e$ but defined over different fields. It is a consequence of a type of modular reduction.

Theorem 1.13 ([14, Theorem 6.35]). Suppose $B$ is a block of $\mathcal{H}_{\mathbb{F}, q}\left(\mathfrak{S}_{n}\right)$, with ecore $\gamma$. Let $q^{\prime}$ be a primitive eth root of unity in $\mathbb{C}$, and let $B_{0}$ be the block of $\mathcal{H}_{\mathbb{C}, q^{\prime}}\left(\mathfrak{S}_{n}\right)$ with e-core $\gamma$.

Let $\mathrm{D}$ and $\mathrm{D}_{0}$ be the decomposition matrices of $B$ and $B_{0}$, respectively, with rows indexed by partitions of $n$ with e-core $\gamma$, and columns indexed by e-regular partitions of $n$ with e-core $\gamma$. Then there exists a square matrix A with non-negative integer entries and with rows and columns both indexed by e-regular partitions of $n$ with e-core $\gamma$, such that $\mathrm{D}=\mathrm{D}_{0} \mathrm{~A}$.

The matrix A in Theorem 1.13 is known as the adjustment matrix for B. Adjustment matrices were introduced by James in [7]; James's Conjecture asserts that if $\operatorname{char}(\mathbb{F})>w$, then the adjustment matrix for a block of $\mathcal{H}_{n}$ of weight $w$ is the identity matrix.

\section{BLOCKS OF SMALL WEIGHT}

In this section, we give some basic results on blocks of weight at most 3 . These are largely concerned with comparing the decomposition numbers for blocks forming a $[3: \kappa]$-pair. The results are largely the same as those in [13], but we are able to give quicker proofs using the modular branching rules.

To begin with, we review the theory of blocks of weight less than 3 . 
2.1. Blocks of weight at most 2. Blocks of weight 0 are simple; thus each contains a unique partition $\nu$, with $S^{\nu}=D^{\nu}$. Blocks of weight 1 are very well understood; each contains $e$ partitions, which may be labelled $\lambda^{1}, \ldots, \lambda^{e}$ so that $\lambda^{1} \triangleright \cdots \triangleright \lambda^{e}$ and that $\lambda^{1}, \ldots, \lambda^{e-1}$ are $e$-regular. The decomposition number $\left[S^{\lambda^{i}}: D^{\lambda^{j}}\right]$ equals 1 if $i=j$ or $j+1$, and 0 otherwise.

Blocks of weight 2 were studied by Richards [16], whose main result we state below; although this was stated only for symmetric group blocks, the proof of the $q$-analogue of the Jantzen-Schaper formula means that it is true in general.

Given a partition $\lambda$ of weight 2 , we reach the core of $\lambda$ by twice moving a bead up one space on the abacus. This corresponds to removing two rim hooks of length $e$ from the Young diagram $[\lambda]$. We denote by $\partial \lambda$ the absolute difference between the leg lengths of these rim hooks. We then have the following.

Theorem 2.1 ([16, Theorem 4.4]). Suppose that $\operatorname{char}(\mathbb{F}) \neq 2$, and that $B$ is a block of $\mathcal{H}_{n}$ of weight 2 . If $\lambda$ and $\mu$ are partitions in $B$ with $\mu$ e-regular, then

$$
\left[S^{\lambda}: D^{\mu}\right]= \begin{cases}1 & (\lambda=\mu), \\ 1 & \left(\lambda=\mu^{\diamond \prime}\right), \\ 1 & \left(\mu \triangleright \lambda \triangleright \mu^{\diamond \prime} \text { and }|\partial \lambda-\partial \mu|=1\right), \\ 0 & \text { (otherwise). }\end{cases}
$$

Corollary 2.2. Suppose $B$ is a block of $\mathcal{H}_{n}$ of weight 2, and that $\lambda, \mu$ and $\nu$ are partitions in $B$ with $\nu$ e-regular. Suppose $\lambda>\mu$ in the lexicographic order, and that $|\partial \lambda-\partial \mu|=1$. If $\left[S^{\lambda}: D^{\nu}\right]=\left[S^{\mu}: D^{\nu}\right]=1$, then either $\nu=\lambda$ or $\nu^{\diamond \prime}=\mu$.

Remark. Theorem 2.1 is not true in characteristic 2 ; the decomposition numbers in this case have been found by the present author [4], but we shall not need these results in this paper.

2.2. Notation for blocks of weight 3. In this section we define some notation for partitions in blocks of weight 3 ; this is similar to the notation used by Martin and Russell [13], but we use the numbering of runners described in \$1.1.6.

Suppose $B$ is a block of $\mathcal{H}_{n}$ of weight 3 , and fix an abacus for $B$. Suppose there are $b_{1}$ beads on the leftmost runner, $b_{2}$ beads on the next runner, and so on, with $b_{e}$ beads on the rightmost runner. Then the $\left\langle b_{1}, \ldots, b_{e}\right\rangle$ notation for the partition $\lambda$ in $B$ is defined as follows. If the display for $\lambda$ is obtained from the display for the core of $B$ by moving the lowest bead on runner $i$ down three spaces, we denote $\lambda$ as $[i]$. If the display for $\lambda$ is obtained by moving the lowest bead down two spaces on runner $i$ and moving a bead down one space on runner $j$ (where $i$ may equal $j$ ), we denote $\lambda$ as $[i, j]$. If the display for $\lambda$ is obtained by moving three beads down one space each on runners $i, j$ and $k$ (where $i, j$ and $k$ may coincide), then we denote $\lambda$ as $[i, j, k]$. In order to emphasise the block in which our partition lies and the abacus used for that block, we may write $[i, j, k]$ as

$$
\left[i, j, k \mid b_{1}, \ldots, b_{e}\right]
$$

and similarly for $[i]$ and $[i, j]$. We may group together equal $b_{i} \mathrm{~s}$; so the partition $\left(4^{2}, 1\right)$ with abacus display

may be written as $\left[1,3 \mid 3^{2}, 2\right]$.

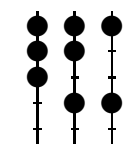


An advantage of using our numbering of the runners of the abacus is that if $A$ and $B$ are blocks forming a $[3: \kappa]$-pair with $\kappa \geq 3$, then the map $\Phi$ described in $\S 1.1 .5$ becomes

$$
\begin{aligned}
{[i, j, k] } & \longmapsto[i, j, k], \\
{[i, j] } & \longmapsto[i, j], \\
{[i] } & \longmapsto[i],
\end{aligned}
$$

for all $i, j, k$.

We make similar definitions for blocks of weight 2 . We write $[i]$ for the partition obtained by moving the lowest bead down two spaces on runner $i$, and $[i, j]$ for the partition obtained by moving two beads down one space each on runners $i$ and $j$. We shall always make the weight of the partition explicit, so no confusion should arise.

2.3. $[3: \kappa]$-pairs. In studying weight 3 blocks, $[3: \kappa]$-pairs are a vital tool. Since blocks forming a $[3: \kappa]$-pair with $\kappa \geq 3$ are Morita equivalent, the study of blocks of weight 3 centres around [3:1]- and [3:2]-pairs. Here we set up some notation and prove some basic results for $[3: \kappa]$-pairs, following Martin and Russell.

Suppose $A \prec B$ form a $[3: \kappa]$-pair, and that the abacus for $B$ is obtained from that for $A$ by swapping the adjacent runners $i$ and $j$, where $i<j$. We say that a partition $\lambda$ in $B$ is exceptional for this $[3: \kappa]$-pair if there are more than $\kappa$ beads on runner $j$ of the abacus display for $B$ with no bead immediately to the left, and non-exceptional otherwise. If $\lambda$ is $e$-regular, then we say that the simple module $D^{\lambda}$ is exceptional if there are more than $\kappa$ normal beads on runner $j$ of the abacus display for $\lambda$. We make similar definitions for $A$ : we say that a partition $\lambda$ in $A$ is exceptional if there are more than $\kappa$ beads on runner $i$ of the abacus display for $\lambda$ with no bead immediately to the right, and if $\lambda$ is $e$-regular we say that $D^{\lambda}$ is exceptional if there are more than $\kappa$ conormal beads on runner $i$.

2.4. [3:1]-pairs. Suppose that $A \prec B$ form a [3:1]-pair, and that the abacus for $B$ is obtained from that for $A$ by swapping runners $i$ and $j$. Then the following are the exceptional partitions in $A$ and $B$ :

A

$$
\begin{aligned}
& \bar{\alpha}_{k}= \begin{cases}{[i, k]} & (k \neq j) \\
{[i]} & (k=j)\end{cases} \\
& \bar{\beta}_{k}= \begin{cases}{[i, j, k]} & (k \neq j) \\
{[j, i]} & (k=j)\end{cases} \\
& \bar{\gamma}_{k}= \begin{cases}{[j, j, k]} & (k \neq i, j) \\
{[j, j, j]} & (k=i) \\
{[j, j]} & (k=j)\end{cases}
\end{aligned}
$$

$B$

$\alpha_{k}= \begin{cases}{[j, j, k]} & (k \neq i, j) \\ {[j, j, j]} & (k=i) \\ {[j, j]} & (k=j)\end{cases}$

$\beta_{k}= \begin{cases}{[i, j, k]} & (k \neq j) \\ {[j, i]} & (k=j)\end{cases}$

$\gamma_{k}= \begin{cases}{[i, k]} & (k \neq j) \\ {[i]} & (k=j) .\end{cases}$

The exceptional simple modules in $A$ and $B$ are the modules $D^{\bar{\alpha}_{k}}$ and $D^{\alpha_{k}}$ for those $k$ such that $\alpha_{k}$ is $e$-regular.

Now we define a bijection between the set of partitions in $B$ and the set of partitions in $A$. If $\lambda$ is a partition in $B$ which is not exceptional, then define the 
partition $\Phi(\lambda)$ in $A$ by swapping runners $j$ and $i$ of the abacus display for $\lambda$. We define $\Phi$ on the exceptional partitions as follows:

$$
\begin{aligned}
& \Phi: \alpha_{k} \longmapsto \bar{\alpha}_{k}, \\
& \beta_{k} \longmapsto \bar{\gamma}_{k}, \\
& \gamma_{k} \longmapsto \bar{\beta}_{k} .
\end{aligned}
$$

The following result is then easily checked.

Lemma 2.3. $\Phi$ is a bijection between the set of partitions in $B$ and the set of partitions in $A$. If $\lambda$ is a partition in $B$, then $\Phi(\lambda)$ is e-regular if and only if $\lambda$ is e-regular.

We get the following results on induction and restriction from Theorems 1.2 and 1.3 .

Proposition 2.4. Suppose that $A$ and $B$ form $a[3: 1]$-pair as above, and that $\lambda$ is a partition in $B$.

- If $\lambda$ is a non-exceptional partition, then

$$
S^{\lambda} \downarrow_{A}^{B} \cong S^{\Phi(\lambda)}, \quad S^{\Phi(\lambda)} \uparrow_{A}^{B} \cong S^{\lambda} .
$$

- If $1 \leq j \leq e$, then

$$
\begin{aligned}
& S^{\alpha_{k}} \downarrow_{A}^{B} \sim S^{\bar{\alpha}_{k}}+S^{\bar{\beta}_{k}}, \quad S^{\bar{\alpha}_{k}} \uparrow_{A}^{B} \sim S^{\alpha_{k}}+S^{\beta_{k}},
\end{aligned}
$$

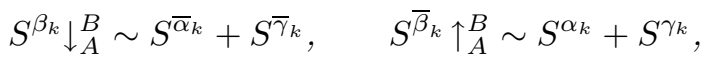

$$
\begin{aligned}
& S^{\gamma_{k}} \downarrow_{A}^{B} \sim S^{\bar{\beta}_{k}}+S^{\bar{\gamma}_{k}}, \quad S^{\bar{\gamma}_{k}} \uparrow_{A}^{B} \sim S^{\beta_{k}}+S^{\gamma_{k}} .
\end{aligned}
$$

- If $\lambda$ is e-regular and $D^{\lambda}$ is a non-exceptional simple module, then

$$
D^{\lambda} \downarrow_{A}^{B} \cong D^{\Phi(\lambda)}, \quad D^{\Phi(\lambda)} \uparrow_{A}^{B} \cong D^{\lambda} .
$$

We now derive some results on the decomposition numbers for blocks forming a [3:1]-pair. Let $A$ and $B$ be as above, and let $C$ be the block of weight 1 whose abacus is obtained from that for $B$ by moving a bead from runner $i$ to runner $j$. We let $\lambda^{1} \triangleright \ldots \triangleright \lambda^{e}$ be the partitions in $C$. We get the following result on induction and restriction between $B$ and $C$ from Theorems 1.2 and 1.3.

Proposition 2.5. Let $B$ and $C$ be as above. Then there is a permutation $\pi \in \mathfrak{S}_{e}$ such that:

(1) if $\lambda$ is a partition in $B$, then

$$
S^{\lambda} \uparrow_{B}^{C} \cong \begin{cases}S^{\lambda^{k}} & \text { (if } \left.\lambda \text { is of the form } \alpha_{\pi(k)}, \beta_{\pi(k)} \text { or } \gamma_{\pi(k)}\right), \\ 0 & \text { (otherwise); }\end{cases}
$$

(2) if $\lambda$ is an e-regular partition in $B$, then

$$
D^{\lambda} \uparrow_{B}^{C} \cong \begin{cases}D^{\lambda^{k}} & \left(\text { if } \lambda \text { is of the form } \alpha_{\pi(k)}\right), \\ 0 & \text { (otherwise). }\end{cases}
$$

Corollary 2.6. Suppose $1 \leq k \leq e-1$. Then $D^{\alpha_{\pi(k)}}$ appears exactly once as a composition factor of each of

$$
S^{\alpha_{\pi(k)}}, \quad S^{\beta_{\pi(k)}}, \quad S^{\gamma_{\pi(k)}}, \quad S^{\alpha_{\pi(k+1)}}, \quad S^{\beta_{\pi(k+1)}}, \quad S^{\gamma_{\pi(k+1)}},
$$

and does not appear as a composition factor of any other Specht module. 
Proof. This follows at once from Proposition 2.5, the decomposition matrix of $C$ described in Section 2.1, and the fact that induction is an exact functor.

As a consequence of this corollary (or by examining the dominance order directly), we see that the partitions $\alpha_{k}$ are totally ordered by dominance, with

$$
\alpha_{\pi(1)} \triangleright \ldots \triangleright \alpha_{\pi(e)} .
$$

Using the weight 1 block obtained from $A$ by moving a bead from runner $j$ to runner $i$, we obtain the following.

Proposition 2.7. Suppose $1 \leq k \leq e-1$. Then $D^{\bar{\alpha}_{\pi(k)}}$ appears exactly once as a composition factor of each of

$$
S^{\bar{\alpha}_{\pi(k)}}, \quad S^{\bar{\beta}_{\pi(k)}}, \quad S^{\bar{\gamma}_{\pi(k)}}, \quad S^{\bar{\alpha}_{\pi(k+1)}}, \quad S^{\bar{\beta}_{\pi(k+1)}}, \quad S^{\bar{\gamma}_{\pi(k+1)}},
$$

and does not appear as a composition factor of any other Specht module.

Finally, we seek to compare the decomposition numbers for $A$ and $B$.

\section{Proposition 2.8.}

(1) Suppose $\lambda$ is a non-exceptional partition in $B$ and $D^{\mu}$ is a non-exceptional simple module in $B$. Then

$$
\left[S^{\Phi(\lambda)}: D^{\Phi(\mu)}\right]=\left[S^{\lambda}: D^{\mu}\right] .
$$

(2) Suppose $D^{\mu}$ is a non-exceptional simple module in $B$, and that $1 \leq k \leq e$. Then

$\left[S^{\alpha_{k}}: D^{\mu}\right]+\left[S^{\bar{\gamma}_{k}}: D^{\Phi(\mu)}\right]=\left[S^{\beta_{k}}: D^{\mu}\right]+\left[S^{\bar{\beta}_{k}}: D^{\Phi(\mu)}\right]=\left[S^{\gamma_{k}}: D^{\mu}\right]+\left[S^{\bar{\alpha}_{k}}: D^{\Phi(\mu)}\right]$. Proof.

(1) This follows from Proposition 2.4, Corollary 2.6 and the fact that restriction is an exact functor.

(2) By Proposition 2.4, Corollary 2.6 and the exactness of restriction, we have

$$
\begin{aligned}
& {\left[S^{\bar{\alpha}_{k}}: D^{\Phi(\mu)}\right]+\left[S^{\bar{\beta}_{k}}: D^{\Phi(\mu)}\right]=\left[S^{\alpha_{k}}: D^{\mu}\right]+\left[D^{\alpha_{k}} \downarrow_{A}^{B}: D^{\Phi(\mu)}\right]+\left[D^{\alpha_{k^{\prime}}} \downarrow_{A}^{B}: D^{\Phi(\mu)}\right],} \\
& {\left[S^{\bar{\alpha}_{k}}: D^{\Phi(\mu)}\right]+\left[S^{\bar{\gamma}_{k}}: D^{\Phi(\mu)}\right]=\left[S^{\beta_{k}}: D^{\mu}\right]+\left[D^{\alpha_{k}} \downarrow_{A}^{B}: D^{\Phi(\mu)}\right]+\left[D^{\alpha_{k^{\prime}}} \downarrow_{A}^{B}: D^{\Phi(\mu)}\right],} \\
& {\left[S^{\bar{\beta}_{k}}: D^{\Phi(\mu)}\right]+\left[S^{\bar{\gamma}_{k}}: D^{\Phi(\mu)}\right]=\left[S^{\gamma_{k}}: D^{\mu}\right]+\left[D^{\alpha_{k}} \downarrow_{A}^{B}: D^{\Phi(\mu)}\right]+\left[D^{\alpha_{k^{\prime}}} \downarrow_{A}^{B}: D^{\Phi(\mu)}\right],}
\end{aligned}
$$

where $k^{\prime}=\pi\left(\pi^{-1}(k)-1\right)$; the factor involving $D^{\alpha_{k}}$ should be ignored if $k=\pi(e)$, and the factor involving $D^{\alpha_{k^{\prime}}}$ should be ignored if $k=\pi(1)$. The equalities and the left-hand inequalities follow. The right-hand inequalities are derived from a very similar argument using induction.

2.5. [3:2]-pairs. In this section we review some background on [3:2]-pairs; the notation here is less complex than for [3:1]-pairs.

Suppose $A \prec B$ form a [3:2]-pair, and that an abacus for $B$ is obtained by swapping runners $i$ and $j$ of an abacus for $A$. We use the following notation for the 
exceptional partitions in $A$ and $B$ :

$$
\begin{array}{ll}
A & B \\
\bar{\alpha}=[i] & \alpha=[j, j, j] \\
\bar{\beta}=[i, j] & \beta=[i, j, j] \\
\bar{\gamma}=[i, j, j] & \gamma=[i, j] \\
\bar{\delta}=[j, j, j] & \delta=[i] .
\end{array}
$$

The exceptional simple modules for this [3:2]-pair are $D^{\bar{\alpha}}$ and $D^{\alpha}$.

We define a bijection $\Phi$ between the set of partitions in $B$ and the set of partitions in $A$, as follows. If $\lambda$ is a non-exceptional partition in $B$, we define $\Phi(\lambda)$ by interchanging runners $i-1$ and $i$ of the abacus display for $\lambda$, while for exceptional partitions we define

$$
\begin{aligned}
\Phi: \alpha & \longmapsto \bar{\alpha} \\
\beta & \longmapsto \bar{\delta} \\
\gamma & \longmapsto \bar{\gamma} \\
\delta & \longmapsto \bar{\beta} .
\end{aligned}
$$

Lemma 2.3 then applies in the present context. The following result follows at once from Theorems 1.2 and 1.3.

Proposition 2.9. Suppose $A$ and $B$ are as above, and $\lambda$ is a partition in $B$.

- If $\lambda$ is non-exceptional, then

$$
S^{\lambda} \downarrow_{A}^{B} \sim\left(S^{\Phi(\lambda)}\right)^{2}, \quad S^{\Phi(\lambda)} \uparrow_{A}^{B} \sim\left(S^{\lambda}\right)^{2} .
$$

- For the exceptional partitions, we have

$$
\begin{aligned}
& S^{\alpha} \downarrow_{A}^{B} \sim\left(S^{\bar{\alpha}}\right)^{2}+\left(S^{\bar{\beta}}\right)^{2}+\left(S^{\bar{\gamma}}\right)^{2}, \quad S^{\bar{\alpha}} \uparrow_{A}^{B} \sim\left(S^{\alpha}\right)^{2}+\left(S^{\beta}\right)^{2}+\left(S^{\gamma}\right)^{2}, \\
& S^{\beta} \downarrow_{A}^{B} \sim\left(S^{\bar{\alpha}}\right)^{2}+\left(S^{\bar{\beta}}\right)^{2}+\left(S^{\bar{\delta}}\right)^{2}, \quad S^{\bar{\beta}} \uparrow_{A}^{B} \sim\left(S^{\alpha}\right)^{2}+\left(S^{\beta}\right)^{2}+\left(S^{\delta}\right)^{2}, \\
& S^{\gamma} \downarrow_{A}^{B} \sim\left(S^{\bar{\alpha}}\right)^{2}+\left(S^{\bar{\gamma}}\right)^{2}+\left(S^{\bar{\delta}}\right)^{2}, \quad S^{\bar{\gamma}} \uparrow_{A}^{B} \sim\left(S^{\alpha}\right)^{2}+\left(S^{\gamma}\right)^{2}+\left(S^{\delta}\right)^{2}, \\
& S^{\delta} \downarrow_{A}^{B} \sim\left(S^{\bar{\beta}}\right)^{2}+\left(S^{\bar{\gamma}}\right)^{2}+\left(S^{\bar{\delta}}\right)^{2}, \quad S^{\bar{\delta}} \uparrow_{A}^{B} \sim\left(S^{\beta}\right)^{2}+\left(S^{\gamma}\right)^{2}+\left(S^{\delta}\right)^{2} .
\end{aligned}
$$

- If $\lambda$ is e-regular and $D^{\lambda}$ is a non-exceptional simple module, then

$$
D^{\lambda} \downarrow_{A}^{B} \cong D^{\Phi(\lambda)} \oplus D^{\Phi(\lambda)}, \quad D^{\Phi(\lambda)} \uparrow_{A}^{B} \cong D^{\lambda} \oplus D^{\lambda} .
$$

Now let $C$ be the block of weight 0 whose abacus is obtained from the abacus for $B$ by moving a bead from runner $i$ to runner $j$. Let $\nu$ denote the unique partition in $C$.

\section{Proposition 2.10.}

(1) If $\lambda$ is a partition in $B$, then

$$
S^{\lambda} \uparrow_{B}^{C} \cong \begin{cases}S^{\nu} & (\text { if } \lambda=\alpha, \beta, \gamma \text { or } \delta), \\ 0 & \text { (otherwise) }\end{cases}
$$

if in addition $\lambda$ is e-regular, then

$$
D^{\lambda} \uparrow_{B}^{C} \cong \begin{cases}D^{\nu} & (\lambda=\alpha), \\ 0 & (\lambda \neq \alpha) .\end{cases}
$$


(2) $D^{\alpha}$ appears once as a composition factor of each of $S^{\alpha}, S^{\beta}, S^{\gamma}, S^{\delta}$, and does not appear as a composition factor of any other Specht module. $D^{\bar{\alpha}}$ appears once as a composition factor of each of $S^{\bar{\alpha}}, S^{\bar{\beta}}, S^{\bar{\gamma}}, S^{\bar{\delta}}$, and does not appear as a composition factor of any other Specht module.

(3) For any $\lambda, \mu$ in $B$ with $\lambda$ non-exceptional and $\mu$ e-regular, we have

$$
\left[S^{\lambda}: D^{\mu}\right]=\left[S^{\Phi(\lambda)}: D^{\Phi(\mu)}\right]
$$

(4) For any non-exceptional simple module $D^{\mu}$ in $B$, we have

$$
\begin{aligned}
& {\left[S^{\alpha}: D^{\mu}\right]+\left[S^{\bar{\delta}}: D^{\Phi(\mu)}\right]=\left[S^{\beta}: D^{\mu}\right]+\left[S^{\bar{\gamma}}: D^{\Phi(\mu)}\right] } \\
= & {\left[S^{\gamma}: D^{\mu}\right]+\left[S^{\bar{\beta}}: D^{\Phi(\mu)}\right]=\left[S^{\delta}: D^{\mu}\right]+\left[S^{\bar{\alpha}}: D^{\Phi(\mu)}\right] . }
\end{aligned}
$$

Proof. (1) follows from Theorems 1.2 and 1.3. (2) and (3) then follow from the exactness of induction and restriction (and the fact that $S^{\nu}=D^{\nu}$ ), while (4) is proved similarly to Proposition 2.8(2).

2.6. Rouquier blocks. A special class of blocks of Hecke algebras is particularly well understood. These are defined for all weights, but we shall restrict attention to blocks of weight 3 .

Let $B$ be a block of weight 3 , and let $\left\{{ }_{i} a_{j}\right\}$ be the pyramid for $B$. We say that $B$ is Rouquier if ${ }_{i} 0_{j}$ for all $i, j$. Thus the Rouquier blocks form a single Scopes class; we shall see later that this class is the greatest class with respect to the order $\preccurlyeq$.

The decomposition numbers for Rouquier blocks (of any weight) are known over a field of infinite characteristic [2, 12]. In addition, a recent paper of James, Lyle and Mathas [8] shows that James's Conjecture holds for Rouquier blocks. As a consequence, we have the following.

Theorem 2.11. Suppose $\operatorname{char}(\mathbb{F}) \geq 5$, that $B$ is a weight 3 Rouquier block of $\mathcal{H}_{n}$, and that $\lambda$ and $\mu$ are partitions in $B$ with $\mu$ e-regular. Then $\left[S^{\lambda}: D^{\mu}\right] \leq 1$.

Proof. If $\operatorname{char}(\mathbb{F})=\infty$, then it easy to read from the explicit combinatorial description of the decomposition numbers ([12, Corollary 10] or [2, Theorem 1.1]) that the decomposition numbers are at most 1 . The general case follows from $[8$, Corollary 4].

2.7. Lowerable partitions. Here we prove a simple lemma which will be in useful in this section and in the next. Suppose $B$ is a weight 3 block of $\mathcal{H}_{n}$, and that in an abacus display for $B$, runner $j$ lies immediately to the right of runner $i$, and the number of beads on runner $i$ exceeds the number of beads on runner $j$ by $b$, for $b=0,1$ or 2 . Let $C$ be the block of $\mathcal{H}_{n-1}$ of weight $2-b$ whose abacus is obtained by moving a bead from runner $j$ to runner $i$. We say that an $e$-regular partition $\mu$ in $B$ is lowerable if $D^{\mu} \downarrow_{C}^{B} \neq 0$ for some such $C$.

Lemma 2.12. Suppose $\operatorname{char}(\mathbb{F}) \geq 3$, that $B$ is a weight 3 block of $\mathcal{H}_{n}$, and that $\mu$ is a lowerable e-regular partition in $B$. Then $\left[S^{\lambda}: D^{\mu}\right] \leq 1$ for all partitions $\lambda$ in $B$.

Proof. Let $C$ be such that $D^{\mu} \downarrow_{C}^{B} \neq 0$. By Theorem 1.2, we find that $S^{\lambda} \downarrow_{C}^{B}$ is either zero or isomorphic to a Specht module. So, since restriction is an exact functor, we find that $\left[S^{\lambda}: D^{\mu}\right]$ is either zero or equal to a decomposition number for $C$. But the decomposition numbers for $C$ are known to be at most 1 . 
In Appendix A, we give a classification of partitions which are not lowerable in certain blocks; this will be useful in Sections 3 and 4. Suppose $e \geq 5$ and that $B$ is the weight 3 block of $\mathcal{H}_{n}$ with core $\left(x^{z}\right)$, where $x$ and $z$ are positive integers with $x+z \leq e$. We let $y=e-x-z$, and use the $\left\langle 3^{x}, 4^{z}, 3^{y}\right\rangle$ abacus notation for partitions in $B$. Table 1 lists all those $e$-regular partitions $\mu$ in $B$ which are not lowerable. For each of these the partition $\mu^{\diamond \prime}$ is calculated. There are fifty cases, each labelled with a pair of letters. The labelling is chosen to reflect the Mullineux map: the conjugate block $B^{\sharp}$ to $B$ has core $\left(z^{x}\right)$, and the non-lowerable partitions in $B^{\sharp}$ may be read off from Table 1 by interchanging $x$ and $z$ throughout. For a partition $\mu$ appearing in Table 1 , the partition $\mu^{\diamond}$ in $B^{\sharp}$ may be found by interchanging the two letters labelling $\mu$ and interchanging $x$ and $z$. For example, we have

$$
\left[x+y+1 \mid 3^{x}, 4^{2}\right]^{\diamond}=\left[1, z+y+1 \mid 3^{2}, 4^{x}\right],
$$

so that case $\mathrm{A}_{\mathrm{E}}$ corresponds to case $\mathrm{E}_{\mathrm{A}}$ under the Mullineux map.

\section{THE CASE $\operatorname{char}(\mathbb{F})=\infty$}

In this section, we prove Theorem 1.1 in the case where $\mathbb{F}$ has infinite characteristic. Using Theorem 1.12, we proceed by induction on $e$, and for given $e$, we use a reverse induction using the partial order $\preccurlyeq$ on the set of Scopes classes of weight 3 blocks, with the Rouquier blocks as a base case. This requires some understanding of how Scopes classes are related, and we define what it means for two Scopes classes to form a $[3: 1]$ - or $[3: 2]$-pair.

Suppose that $\mathcal{C}, \mathcal{D}$ are Scopes classes, that $\mathcal{C} \prec \mathcal{D}$ and that $A$ and $B$ are blocks forming a $[3: 2]$-pair, with $A \in \mathcal{C}$ and $B \in \mathcal{D}$. Suppose moreover that the abacus for $B$ is obtained from that for $A$ by moving two beads from runner $j$ to runner $i$ (so $i<j$ ). The exceptional partitions in $A$ for this [3:2]-pair are then $[i],[i, j],[i, j, j]$ and $[j, j, j]$, and so, by Proposition 2.8, every decomposition number $\left[S^{\lambda}: D^{\mu}\right]$ for $A$ can be equated with a decomposition number for $B$ as long as $\lambda$ is not one of these four partitions. Hence for any block $A^{\prime}$ in $\mathcal{C}$, the decomposition number $\left[S^{\lambda}: D^{\mu}\right]$ can be equated with a decomposition number for $B$, as long as $\lambda$ does not equal $[i]$, $[i, j],[i, j, j]$ or $[j, j, j]$. We say that $\mathcal{C}$ and $\mathcal{D}$ form $a[3: 2]$-pair on runners $i$ and $j$. Analogously, we define what it means for $\mathcal{C}$ and $\mathcal{D}$ to form a $[3: 1]$-pair on runners $i$ and $j$; here there are more exceptional partitions, but they are easily listed, as in Section 2.4.

Our technique in proving Theorem 1.1 for fields of infinite characteristic is to suppose that $B$ lies in a Scopes class $\mathcal{C}$, and that Theorem 1.1 holds for all blocks in Scopes classes $\mathcal{D} \succ \mathcal{C}$. We then examine with which classes $\mathcal{C}$ can form a [3:1]- or $[3: 2]$-pair; in most cases, we find that we can equate each decomposition number for a block in $\mathcal{C}$ with a decomposition number for a block in some such $\mathcal{D}$. We must then deal with the remaining cases.

To find [3:2]- and [3:1]-pairs between Scopes classes, we examine their pyramids; recall the definition of ${ }_{i} a_{j}$ from Section 1.1.6.

Lemma 3.1. Suppose $\mathcal{C}$ is a Scopes class, and that $1 \leq i<j \leq e$.

(1) There is a Scopes class $\mathcal{D} \succ \mathcal{C}$ such that $\mathcal{C}$ and $\mathcal{D}$ form a [3:1]-pair on runners $i$ and $j$ if and only if

(a) ${ }_{i} 2_{j}$,

(b) there is no $k<i$ such that ${ }_{k} a_{i}={ }_{k} a_{j}>0$, and

(c) there is no $k>j$ such that ${ }_{i} a_{k}={ }_{j} a_{k}>0$. 
(2) There is a Scopes class $\mathcal{D} \succ \mathcal{C}$ such that $\mathcal{C}$ and $\mathcal{D}$ form a [3:2]-pair on runners $i$ and $j$ if and only if

(a) ${ }_{i} 1_{j}$,

(b) there is no $k<i$ with ${ }_{k} 1_{j}$,

(c) there is no $i<k<j$ with ${ }_{i} 2_{k} 2_{j}$, and

(d) there is no $k>j$ with ${ }_{i} 1_{k}$.

Proof. We prove (1); the proof of (2) is very similar.

Suppose $A$ and $B$ form a $[3: 1]$-pair for $A \in \mathcal{C}, B \in \mathcal{D}$ on runners $i$ and $j$. Choose an abacus display for $A$ so that runners $i$ and $j$ are adjacent (with runner $i$ to the right of runner $j$ ). Then there must be one more bead on runner $j$ than on runner $i$, so we have ${ }_{i} 2_{j}$. If $k$ is as in (1b) or (1c), then runner $k$ must lie between runners $j$ and $i$, which it cannot.

Conversely, suppose that the pyramid for $\mathcal{C}$ satisfies the conditions given, and take an abacus display for some block in $\mathcal{C}$ in which runner $i$ is to the right of runner $j$. Suppose there are $a$ beads on runner $i$; then, since ${ }_{i} 2_{j}$, there must be $a+1$ beads on runner $j$; furthermore, if runner $k$ lies between runners $j$ and $i$, then the number of beads on runner $k$ is either at most $a-2$ or at least $a+3$. If there is a runner between $i$ and $j$ with at least $a+3$ beads, let runner $k$ be the rightmost such. Then runner $k$ has at least three more beads than runner $i$ or any runner between $k$ and $i$. So we may successively swap runner $k$ with these runners, and we reach, via a sequence of $[3: \kappa]$-pairs with $\kappa \geq 3$, a block $A^{\prime}$ in $\mathcal{C}$ with fewer runners between $j$ and $i$. Similarly, we move any runner with at most $a-2$ beads to the left. In this way, we can reach a block in $\mathcal{C}$ with no runners between $j$ and $i$, which therefore forms a $[3: 1]$-pair on these runners.

Corollary 3.2. Suppose $\mathcal{C}, \mathcal{D}_{1}, \mathcal{D}_{2}$ are distinct Scopes classes such that, for $l=1,2$ :

- $\mathcal{C} \prec \mathcal{D}_{l}$;

- $\mathcal{C}$ and $\mathcal{D}_{l}$ form a $\left[3: \kappa_{l}\right]$-pair on runners $i_{l}$ and $j_{l}$, where $\kappa_{l}=1$ or 2.

Then $i_{1} \neq i_{2}$ and $j_{1} \neq j_{2}$.

Proof. The pyramid for $\mathcal{D}_{l}$ is obtained from that for $\mathcal{C}$ by increasing $i_{l} a_{j_{l}}$ by 1 . In particular, $\mathcal{C}, i_{l}$ and $j_{l}$ determine $\mathcal{D}_{l}$, and so we cannot have $i_{1}=i_{2}$ and $j_{1}=j_{2}$. So suppose that $i_{1}=i_{2}$ and $j_{1}<j_{2}$. Since $\kappa_{l}=3-{ }_{i_{l}} a_{j_{l}}$, this implies that $\kappa_{1} \leq \kappa_{2}$. Furthermore, the conditions of Lemma 3.1 imply that $j_{l}$ is maximal such that $\kappa_{l}=3-{ }_{i_{l}} a_{j_{l}}$; so we cannot have $\kappa_{1}=\kappa_{2}$.

So $\kappa_{l}=l$ for $l=1,2$. The conditions for the [3:2]-pair imply that $i_{1} a_{j_{2}}=$ $j_{1} a_{j_{2}}=1$, but the conditions for the [3:1]-pair say that there is no such $j_{2}$.

A similar argument applies when $i_{1}<i_{2}$ and $j_{1}=j_{2}$.

In view of this, we can find several circumstances where each decomposition number for a block in $\mathcal{C}$ can be equated with a decomposition number for a block in a higher class.

Lemma 3.3. Suppose that $\mathcal{C}, \mathcal{D}_{1}, \mathcal{D}_{2}$ are distinct Scopes classes such that, for $l=$ 1,2 :

- $\mathcal{C} \prec \mathcal{D}_{l}$;

- $\mathcal{C}$ and $\mathcal{D}_{l}$ form a $\left[3: \kappa_{l}\right]$-pair on runners $i_{l}$ and $j_{l}$, where $\kappa_{l}=1$ or 2 .

Suppose also that if $\kappa_{1}=\kappa_{2}=1$, then $i_{1} \neq j_{2}$ and $i_{2} \neq j_{1}$.

Then every decomposition number for a block in $\mathcal{C}$ can be equated with a decomposition number for a block in either $\mathcal{D}_{1}$ or $\mathcal{D}_{2}$. 
Proof. By the above discussion, we know that each decomposition number $\left[S^{\lambda}: D^{\mu}\right]$ for a block in $\mathcal{C}$ can be equated to a decomposition number for a block in $\mathcal{D}_{l}$ unless $\lambda \in \Lambda_{1} \cap \Lambda_{2}$, where $\Lambda_{l}$ equals

$$
\begin{cases}\left\{\left[i_{l}\right],\left[j_{l}, i_{l}\right],\left[j_{l}, j_{l}\right]\right\} \cup\left\{\left[i_{l}, m\right],\left[i_{l}, j_{l}, m\right] \mid m \neq j_{l}\right\} \cup\left\{\left[j_{l}, j_{l}, m\right] \mid m \neq i_{l}\right\} & \left(\kappa_{l}=1\right), \\ \left\{\left[i_{l}\right],\left[i_{l}, j_{l}\right],\left[i_{l}, j_{l}, j_{l}\right],\left[j_{l}, j_{l}, j_{l}\right]\right\} & \left(\kappa_{l}=2\right) .\end{cases}
$$

The conditions given for $i_{l}, j_{l}$ imply that $\Lambda_{1}$ and $\Lambda_{2}$ are disjoint.

So the only cases where there are some decomposition numbers for blocks in $\mathcal{C}$ which cannot be equated with decomposition numbers for higher classes are as follows.

(C1) There is no Scopes class $\mathcal{D} \succ \mathcal{C}$ with which $\mathcal{C}$ forms a [3:1]- or [3:2]-pair.

(C2) There is exactly one Scopes class $\mathcal{D} \succ \mathcal{C}$ with which $\mathcal{C}$ forms a [3:1]-pair, and no $\mathcal{D}$ with which $\mathcal{C}$ forms a $[3: 2]$-pair.

(C3) There are two Scopes classes $\mathcal{D}_{1}, \mathcal{D}_{2} \succ \mathcal{C}$ and $1 \leq i<j<k \leq e$ such that $\mathcal{C}$ and $\mathcal{D}_{1}$ form a $[3: 1]$-pair on runners $i$ and $j$, while $\mathcal{C}$ and $\mathcal{D}_{2}$ form a $[3: 1]$-pair on runners $j$ and $k$. There are no other classes $\mathcal{D}$ with which $\mathcal{C}$ forms a $[3: 1]$ - or $[3: 2]$-pair.

(C4) There is exactly one Scopes class $\mathcal{D} \succ \mathcal{C}$ with which $\mathcal{C}$ forms a [3:2]-pair, and no $\mathcal{D}$ with which $\mathcal{C}$ forms a $[3: 1]$-pair.

To prove Theorem 1.1, we must study these four cases. First, we describe all the corresponding Scopes classes in terms of pyramids.

Lemma 3.4. Suppose $\mathcal{C}$ is a Scopes class of weight 3 blocks, with pyramid $\left\{{ }_{i} a_{j} \mid\right.$ $1 \leq i<j \leq e\}$.

(1) $\mathcal{C}$ satisfies condition $\mathrm{C} 1$ above if and only if ${ }_{i} 0_{j}$ for all $i, j$.

(2) $\mathcal{C}$ satisfies condition $\mathrm{C} 2$ above if and only if there exist $i<j$ such that

- ${ }_{i} 2_{j}$, and

- ${ }_{k} 0_{l}$ whenever $k<i$ or $l>j$.

(3) $\mathcal{C}$ satisfies condition $\mathrm{C} 3$ above if and only if

- ${ }_{i} 2_{j} 2_{k}$,

- ${ }_{l} 1_{m}$ whenever $i \leq l<j<m \leq k$, and

- ${ }_{l} 0_{m}$ whenever $l<i$ or $m>k$.

(4) $\mathcal{C}$ satisfies condition $\mathrm{C} 4$ above if and only if there exist $i<k<k+1<j$ such that

- ${ }_{i} 1_{j}$,

- ${ }_{k} 1_{k+1}$, and

- ${ }_{l} 0_{m}$ whenever $l<i$ or $m>j$.

Proof. In each case the 'if' condition is easily verified; in cases C2 and C4 the $[3: \kappa]$-pair in question is on runners $i$ and $j$.

For the 'only if' parts, we suppose that the pyramid for $\mathcal{C}$ does not satisfy the conditions given in any of (1)-(4). Define a pair $(i, j)$ with $1 \leq i<j \leq e$ to be a peak if

$$
0={ }_{i-1} a_{j}={ }_{i} a_{j+1}<{ }_{i} a_{j}
$$


Note that if $(i, j)$ and $\left(i^{\prime}, j^{\prime}\right)$ are peaks with $i \leq i^{\prime}$, then $i<i^{\prime}$ and $j<j^{\prime}$. We say in this case that $(i, j)$ is smaller than $\left(i^{\prime}, j^{\prime}\right)$.

Suppose that there is at least one peak, and that $(i, j)$ is the smallest peak. We claim that there is some $k \leq j$ such that $\mathcal{C}$ forms either a [3:1]- or a [3:2]-pair on runners $i$ and $k$. If ${ }_{i} 2_{j}$, then $\mathcal{C}$ forms a [3:1]-pair on $i$ and $j$, so suppose ${ }_{i} 1_{j}$. If there is no $i<l<j$ such that ${ }_{i} 2_{l} 2_{j}$, then $\mathcal{C}$ forms a [3:2]-pair on runners $i$ and $j$. If there is such an $l$, let $k$ be the maximal such; $k$ is then maximal such that ${ }_{i} 2_{k}$. Since $(i, j)$ is the smallest peak, we have ${ }_{i-1} 0_{k}$, and so we find that $\mathcal{C}$ forms a [3:1]-pair on runners $i$ and $k$.

Similarly, if $\left(i^{\prime}, j^{\prime}\right)$ is the largest peak, then $\mathcal{C}$ forms a $[3: 1]$ - or [3:2]-pair on runners $k^{\prime}$ and $j^{\prime}$ for some $k^{\prime} \geq i^{\prime}$.

If there are at least two peaks, let $(i, j)$ and $\left(i^{\prime}, j^{\prime}\right)$ be the smallest and largest. Then $\mathcal{C}$ forms [3:1]- or [3:2]-pairs on $(i, k)$ and $\left(k^{\prime}, j^{\prime}\right)$ for some $k, k^{\prime}$; the only way we could then be in any of the cases $(\mathrm{C} 1)-(\mathrm{C} 4)$ is if both the pairs are [3:1]-pairs and $k=k^{\prime}$. But then we would have ${ }_{i} 2_{k} 2_{j^{\prime}}$ and (since $(i, j)$ and $\left(i^{\prime}, j^{\prime}\right)$ are distinct peaks) ${ }_{i} 0_{j^{\prime}}$; this is not possible.

So we may assume that there is exactly one peak, at $(i, j)$. (If there are no peaks, then ${ }_{i} 0_{j}$ for all $i, j$, so the pyramid is as described in (1).) By assumption we cannot have ${ }_{i} 2_{j}$ (since then the pyramid would be as in (2)), and we cannot have ${ }_{k} 1_{k+1}$ for any $k$ (or the pyramid would be as in (4)). Let $l$ be minimal such that ${ }_{i} 1_{l}$, let $i^{\prime}$ be minimal such that ${ }_{i^{\prime}} 2_{l}$, and let $j^{\prime}$ be maximal such that ${ }_{i^{\prime}} 2_{j^{\prime}}$. Then $\mathcal{C}$ forms a [3:1]-pair on $\left(i^{\prime}, j^{\prime}\right)$, so we cannot be in case $\mathrm{C} 4$; so $\mathcal{C}$ does not form a $[3: 2]$-pair on $(i, j)$, and hence there is some $k$ such that ${ }_{i} 2_{k} 2_{j}$. This means that $\mathcal{C}$ forms a $[3: 1]$-pair on $(m, n)$ for every pair $(m, n)$ such that

$$
2={ }_{m} a_{n}>_{m-1} a_{n},{ }_{m} a_{n+1} .
$$

There are at least two such pairs, so if we are in one of the cases $(\mathrm{C} 1)-(\mathrm{C} 4)$, then there must be exactly two such pairs, and they must be $(i, k)$ and $(k, j)$ for some $k$. But then the pyramid is as described in (3).

In order to prove Theorem 1.1, we assume that $\mathcal{C}$ is in one of the cases $(\mathrm{C} 1)-(\mathrm{C} 4)$ and that the decomposition numbers for any class $\mathcal{D} \succ \mathcal{C}$ are at most 1 . We must then prove that the decomposition numbers in $\mathcal{C}$ are at most 1.

Case $\mathrm{C} 1$ is dealt with by Theorem 2.11 , so we turn our attention to the other cases.

3.1. Case C2. The main result of this subsection is the following.

Proposition 3.5. Suppose that $\mathbb{F}$ has infinite characteristic, and that $e \geq 5$. Suppose also that $B$ is a weight 3 block in a Scopes class $\mathcal{C}$ which forms exactly one [3:1]-pair with a Scopes class $\mathcal{D}$, and no [3:2]-pairs. If the decomposition numbers for blocks in $\mathcal{D}$ are all at most 1 and Theorem 1.1 holds over $\mathbb{F}$ with e replaced by $e-1$, then the decomposition numbers for $B$ are all at most 1 .

First we need a lemma describing the map $\mu \mapsto \mu^{\diamond \prime}$ for certain partitions in certain blocks. We assume throughout this subsection that $e \geq 5$.

Lemma 3.6. Suppose that $1 \leq c \leq e-1$, and that $B_{c}$ is the weight 3 block of $\mathcal{H}_{n}$ with the $\left\langle 3^{c}, 5,7, \ldots, 2(e-c)+3\right\rangle$ notation. 
Then in $B_{c}$ we have

$$
\begin{aligned}
& {[e]^{\diamond \prime}=} \begin{cases}{[e-1, e-1, e-1]} & (c \leq e-2), \\
{[e-1, e-2, e-3]} & (c=e-1),\end{cases} \\
& {[e, e]^{\diamond \prime}= \begin{cases}{[e-1, e-1]} & (c \leq e-2), \\
{[e-1, e-2]} & (c=e-1),\end{cases} } \\
& {[e, i]^{\diamond \prime}= \begin{cases}{[e-1, e-1, i-1]} & (2 \leq i \leq e-1, c \leq e-2), \\
{[i-1, e-1]} & (2 \leq i \leq e-1, c=e-1) .\end{cases} }
\end{aligned}
$$

Proof. The case $c=e-1$ may be dealt with directly by using Mullineux's algorithm. When $c \leq e-2$, the result may be read off from [2, Theorem 1.1]; the partitions in the lemma lie in the set $\mathcal{P}_{\kappa}$ described in that paper, where the decomposition numbers $\left[S^{\lambda}: D^{\mu}\right]$ are described for $\mu \in \mathcal{P}_{\kappa}$. For any $e$-regular $\mu$, the partition $\mu^{\diamond \prime}$ is the least dominant partition $\lambda$ for which $\left[S^{\lambda}: D^{\mu}\right]>0$, and so is easily obtained.

Proof of Proposition 3.5. By Lemma 3.4 we may deduce the form of the pyramid for $\mathcal{C}$, and hence the abacus for some block in $\mathcal{C}$. So without loss of generality we may assume that there exist $a, b \geq 0$ and $c \geq 2$ with $a+b+c=e$ and that $B$ is the block with the

$$
\left\langle 3,5,7, \ldots, 1+2 a,(3+2 a)^{c}, 5+2 a, 7+2 a, \ldots, 3+2 a+2 b\right\rangle
$$

notation. This abacus may be pictured as follows:

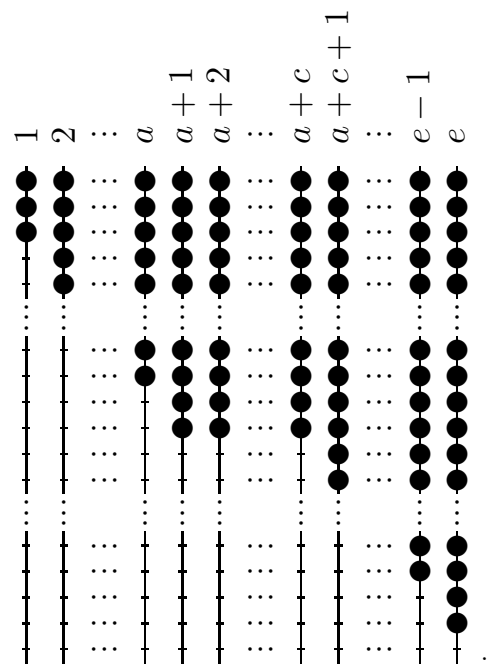

Since by assumption all the decomposition numbers for blocks in $\mathcal{D}$ are at most 1 , the same is true for the decomposition numbers $\left[S^{\lambda}: D^{\mu}\right]$ in $B$, except possibly 
when $\lambda$ is one of the following partitions (which we label analogously with Section 2.4):

$$
\begin{aligned}
& \bar{\alpha}_{k}= \begin{cases}{[a+1, k]} & (k \neq a+c), \\
{[a+1]} & (k=a+c) ;\end{cases} \\
& \bar{\beta}_{k}= \begin{cases}{[a+1, a+c, k]} & (k \neq a+c), \\
{[a+c, a+1]} & (k=a+c) ;\end{cases} \\
& \bar{\gamma}_{k}= \begin{cases}{[a+c, a+c, k]} & (k \neq a+1, a+c), \\
{[a+c, a+c, a+c]} & (k=a+1), \\
{[a+c, a+c]} & (k=a+c) .\end{cases}
\end{aligned}
$$

So for the remainder of the proof we assume that $\lambda$ is one of these partitions and that $\mu$ is an $e$-regular partition with $\mu \unrhd \lambda \unrhd \mu^{\diamond \prime}$. Furthermore, if $\lambda=\bar{\alpha}_{k}, \bar{\beta}_{k}$ or $\bar{\gamma}_{k}$, then we may assume that $\mu \unrhd \bar{\alpha}_{k}$ and $\bar{\gamma}_{k} \unrhd \mu^{\diamond \prime}$, since by Proposition 2.8(2) we find that if $\left[S^{\lambda}: D^{\mu}\right] \geq 2$, then $\left[S^{\bar{\alpha}_{k}}: D^{\mu}\right],\left[S^{\bar{\gamma}_{k}}: D^{\mu}\right] \geq 1$. By Corollary 2.6, we may also assume that $\mu$ does not equal $\bar{\alpha}_{i}$ for any $i$.

If $a \geq 1$ and $k \geq 2$, then the assumption $\mu \triangleright \bar{\alpha}_{k}$ means that $\lambda$ and $\mu$ can be displayed on an abacus with an empty runner, namely the same abacus as above but with three beads removed from each runner. If we define $\lambda^{-}$and $\mu^{-}$to be the partitions obtained by removing this runner (as in Section 1.1.8) and if $\mu^{-}$is $(e-1)$ regular, then by Theorem 1.12 we may equate the decomposition number $\left[S^{\lambda}: D^{\mu}\right]$ with a decomposition number for a weight 3 block of an Iwahori-Hecke algebra at an $(e-1)$ th root of unity; by our inductive assumption this decomposition number will be either 0 or 1 . So we assume for the rest of the proof that either $a=0$ or $k=1$ or $\mu^{-}$is $(e-1)$-singular. We consider several cases.

$[a=b=0]:$ In this case, it is easy to check that $\mu$ is always lowerable. So the proposition holds here by Lemma 2.12 .

$[a \geq 2, k \geq 2]:$ In this case, the conditions that $\mu \triangleright \bar{\alpha}_{2}$ and $\mu^{-}$is $(e-1)$ singular imply that $a=k=2$ and that the first two runners of the abacus display for $\mu$ have the form

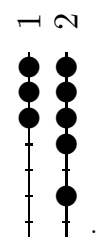

Then we find that for $i=1,2,3$ we have $\mu_{i}^{\prime}=\lambda_{i}^{\prime}$. So we may apply Theorem 1.11; we define

$\bar{\lambda}=\left(\max \left(\lambda_{1}-3,0\right), \max \left(\lambda_{2}-3,0\right), \ldots\right), \quad \bar{\mu}=\left(\max \left(\mu_{1}-3,0\right), \max \left(\mu_{2}-3,0\right), \ldots\right)$.

Then we have

$$
\left[S^{\lambda}: D^{\mu}\right]=\left[S^{\bar{\lambda}}: D^{\bar{\mu}}\right]
$$

and this is at most 1 , since $\bar{\lambda}$ and $\bar{\mu}$ are partitions of weight 2 .

$[a=1, b=0, k \geq 2]:$ The conditions that $\mu \triangleright \bar{\alpha}_{k}$ and that $\mu^{-}$is $(e-1)-$ singular imply that $\mu=[i, i]$ for some $i \geq 2$. Furthermore, we cannot have $\mu=[2,2]=\bar{\alpha}_{e}$. But if $i \geq 3$, then $\mu$ is lowerable, and so $\left[S^{\lambda}: D^{\mu}\right] \leq 1$ by Lemma 2.12. 
$[a=1, b=1, k \geq 2]$ : We assume that $\mu$ is not lowerable. Together with our other assumptions on $\mu$, this implies that $\mu$ is one of the four partitions

$$
[e, 2], \quad[e, 3,2], \quad[e, e, 2], \quad[e, 2,2] .
$$

We may analyse these using the Jantzen-Schaper formula. First we apply Mullineux's algorithm to find that

$$
[e, 2]^{\diamond}=[e, 3,2], \quad[e, e, 2]^{\diamond}=[e, 2,2] .
$$

Now we examine the cases $\mu=[e, 2]$ and $\mu=[e, e, 2]$ explicitly; see Appen$\operatorname{dix}$ B. The other two cases follow using Corollary 1.5.

$[a=0, b \geq 1, k=e]$ : Since $\mu \unrhd \lambda, \mu$ must have a bead of weight at least 1 on runner $e$. If the lowest bead on runner $e$ has weight exactly 1 , then $\lambda$ and $\mu$ have the same first part, and so we may apply Theorem 1.11: we have $\left[S^{\lambda}: D^{\mu}\right]=\left[S^{\hat{\lambda}}: D^{\hat{\mu}}\right]$, where

$$
\hat{\lambda}=\left(\lambda_{2}, \lambda_{3}, \ldots\right), \quad \hat{\mu}=\left(\mu_{2}, \mu_{3}, \ldots\right)
$$

are partitions of weight 2 . Hence by Theorem $1.11(1)$ we have $\left[S^{\lambda}: D^{\mu}\right] \leq 1$.

So we may assume that there is a bead of weight at least 2 on runner $e$ in the abacus display for $\mu$, i.e. $\mu$ is one of the partitions $[e]$ or $[e, i]$ for $1 \leq i \leq e$. But then by Lemma 3.6, $\mu^{\diamond \prime}$ has at most one bead of positive weight on any of the runners $1, \ldots, c$, and so $\bar{\gamma}_{e} \not \mu^{\diamond \prime}$, a contradiction.

$[a=1, b \geq 2, k=e]$ : Since $\mu \unrhd \lambda$, there must be a bead of weight at least 1 on runner $e$ in the abacus display for $\mu$. As above, if the lowest bead on this runner has weight exactly 1 , then we have $\lambda_{1}=\mu_{1}$ and we may appeal to Theorem 1.11(1). So we assume that there is a bead of weight at least 2 on runner $e$ in the abacus display for $\mu$. The condition that $\mu^{-}$is $(e-1)$-singular then implies that $\mu=[e, 2]$. By appealing to [2, Theorem 1.1] as in the proof of Lemma 3.6, we find that

$$
\mu^{\diamond \prime}=[e-1, e-1,1] .
$$

But then $\bar{\gamma}_{e} \not \mu^{\diamond \prime}$, a contradiction.

$[(a \geq 1 \geq b, k=1)$ or $(b \geq 1 \geq a, k \leq e-1)]$ : We replace $B, \lambda, \mu$ with $B^{\sharp}$, $\lambda^{\prime}, \mu^{\diamond}$, and appeal to the previous cases (and Corollary 1.5).

3.2. Case C3. Cases C3 and C4 are rather easier to deal with than Case C2. We prove the following statement for Case C3.

Proposition 3.7. Suppose that $\mathbb{F}$ has infinite characteristic, that $e \geq 5$, and that $B$ is a weight 3 block lying in a Scopes class $\mathcal{C}$. Suppose that there are two Scopes classes $\mathcal{D}_{1}, \mathcal{D}_{2}$ and $1 \leq i<j<k \leq e$ such that $\mathcal{C}$ and $\mathcal{D}_{1}$ form a [3:1]-pair on runners $i$ and $j$, while $\mathcal{C}$ and $\mathcal{D}_{2}$ form $a[3: 1]$-pair on runners $j$ and $k$. Suppose also that there are no other classes $\mathcal{D}$ with which $\mathcal{C}$ forms $a[3: 1]$ - or [3:2]-pair. If the decomposition numbers for blocks in $\mathcal{D}_{1}$ and $\mathcal{D}_{2}$ are all at most 1 and Theorem 1.1 holds over $\mathbb{F}$ with e replaced by $e-1$, then the decomposition numbers for $B$ are all at most 1.

Proof. By Lemma 3.4, we may assume that $B$ is the block with the

$$
\left\langle 3,5,7, \ldots, 1+2 a,(3+2 a)^{c},(4+2 a)^{d}, 6+2 a, 8+2 a, \ldots, 4+2 a+2 b, 3+2 a\right\rangle
$$


notation, where $a+b+c+d=e-1$. (In fact, we have $a=i-1, c=j-i, d=k-j$, $b=e-k$.) The abacus for this block may be pictured as follows:

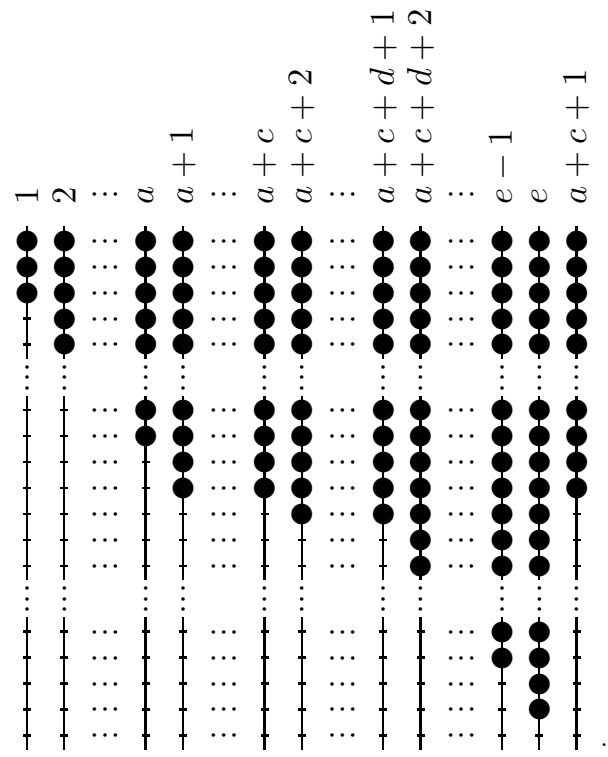

By replacing $B$ with its conjugate block if necessary, we may assume $a \geq b$.

Since we assume that the decomposition numbers for $\mathcal{D}_{1}$ and $\mathcal{D}_{2}$ are at most 1 , the same is true for the decomposition numbers $\left[S^{\lambda}: D^{\mu}\right]$ for $B$, except for those $\lambda$ which are exceptional for both of the $[3: 1]$-pairs. There are four such partitions, which we label as follows:

$$
\begin{array}{llrl}
\overline{\beta \gamma} & =[a+c+1, a+c+1, a+c+d+1], & \overline{\beta \beta} & =[a+c+1, a+c+d+1, a+1], \\
\overline{\alpha \gamma} & =[a+c+1, a+c+1], & \overline{\alpha \beta} & =[a+c+1, a+1] .
\end{array}
$$

We also define the following partitions:

$$
\begin{aligned}
& \overline{\gamma \gamma}=[a+c+d+1, a+c+d+1, a+c+d+1], \\
& \overline{\gamma \beta}=[a+c+d+1, a+c+d+1, a+1], \\
& \overline{\beta \alpha}=[a+1, a+c+d+1], \\
& \overline{\alpha \alpha}=[a+1] .
\end{aligned}
$$

Now suppose $\mu$ is an $e$-regular partition in $B$ with $\left[S^{\lambda}: D^{\mu}\right]>1$ for some $\lambda$. Then we claim that we must have $\mu \triangleright \overline{\beta \alpha}$. For if $\lambda=\overline{\beta \gamma}$ or $\overline{\beta \beta}$, then by Proposition $2.8(2)$ and by our assumption on the decomposition numbers for blocks in $\mathcal{D}_{1}$ we have $\left[S^{\overline{\beta \alpha}}: D^{\mu}\right] \geq 1$, so that $\mu \unrhd \overline{\beta \alpha}$; if $\lambda=\overline{\alpha \gamma}$ or $\overline{\alpha \beta}$, then in the same way we find that $\mu \unrhd \overline{\alpha \alpha} \unrhd \overline{\beta \alpha}$. Furthermore, we cannot have $\mu=\overline{\beta \alpha}$, by Proposition 2.7. By similar arguments, we find that $\mu \triangleright \overline{\alpha \gamma}$, and also that $\overline{\alpha \gamma} \triangleright \mu^{\diamond \prime}$ and $\overline{\gamma \beta} \triangleright \mu^{\diamond \prime}$.

If $a \geq 1$, then $\lambda$ and $\mu$ can both be displayed on an abacus with an empty runner, namely the same abacus as above but with $3 e$ fewer beads. We define $\lambda^{-}$and $\mu^{-}$ to be the partitions obtained by removing this runner, as in Section 1.1.8. If $\mu^{-}$is $(e-1)$-regular, then we may equate $\left[S^{\lambda}: D^{\mu}\right]$ with a decomposition number for a weight 3 block of a Hecke algebra at an $(e-1)$ th root of unity; by our inductive assumption, such a decomposition number is at most 1 . So we are left to consider only those cases where $a=0$ or $\mu^{-}$is $(e-1)$-singular. If $a \geq 2$, then $\mu^{-}$is always 
$(e-1)$-regular, and so we are left with the cases where $a \leq 1$. By Lemma 2.12, we may also assume that $\mu$ is not lowerable. We examine the possibilities for $a$ and $b$.

$[a=b=1]:$ The conditions that $\mu \triangleright \overline{\beta \alpha}, \mu \triangleright \overline{\alpha \gamma}, \mu^{-}$is $(e-1)$-singular and $\mu$ is not lowerable imply that $\mu$ is one of the four partitions $[c+3,2],[e, 2]$, $[e, e, 2],[e, c+3,2]$. For each of these possibilities we calculate $\mu^{\diamond}$ :

\begin{tabular}{c|cc}
\hline$\mu$ & \multicolumn{2}{|c}{$\mu^{{ }^{\prime}}$} \\
\hline$[c+3,2]$ & {$[e-1, c+2,1]$} & $(d>1)$ \\
& {$[c+2, c+1,1]$} & $(d=1)$ \\
\hline$[e, 2]$ & {$[e-1, e-2,1]$} & $(d>1)$ \\
& {$[e-1, c+2,1]$} & $(d=1)$ \\
\hline$[e, e, 2]$ & \multicolumn{2}{|c}{$[e-1,1]$} \\
\hline$[e, c+3,2]$ & {$[c+2,1]$} \\
\hline
\end{tabular}

We find that in none of these cases do we have $\overline{\gamma \beta} \triangleright \mu^{\diamond \prime}$, except the case $\mu=[c+3,2]$ and $d=1$. In this case we look at $\mu^{\diamond}$, which lies in the block conjugate to $B$, and we find that

$$
\mu^{\diamond}=\left[e, 4,3 \mid 3,5,6^{c}, 8,5\right]
$$

so $\left(\mu^{\diamond}\right)^{-}$is $(e-1)$-regular.

$[a=1, b=0]$ : This case is dealt with in the same way as the last one, but here it is much easier. The only partition $\mu$ such that $\mu \triangleright \overline{\beta \alpha}, \mu \triangleright \overline{\alpha \gamma}, \mu^{-}$ is $(e-1)$-singular and $\mu$ is not lowerable is $\mu=[c+3,2]$, for which we have

$$
\mu^{\diamond \prime}= \begin{cases}{[e, c+2,1]} & (d>1), \\ {[c+2, c+1,1]} & (d=1),\end{cases}
$$

and so we do not have $\overline{\gamma \beta} \triangleright \mu^{\diamond \prime}$.

$[a=b=0]$ : Here we examine Table 1 (putting $x=c, y=1, z=d$ ) to find those partitions $\mu$ which are not lowerable and which satisfy $\mu \triangleright \overline{\beta \alpha}$ and $\mu \triangleright \overline{\alpha \gamma}$. We find that these correspond to cases $\mathrm{A}_{\mathrm{H}}, \mathrm{A}_{\mathrm{K}}, \mathrm{A}_{\mathrm{N}}, \mathrm{B}_{\mathrm{G}}, \mathrm{C}_{\mathrm{H}}, \mathrm{C}_{\mathrm{N}}$ and $\mathrm{D}_{\mathrm{F}}$. But in none of these cases do we have $\overline{\alpha \gamma} \triangleright \mu^{\diamond}$.

\subsection{Case C4.}

Proposition 3.8. Suppose $\mathbb{F}$ has infinite characteristic, that $e \geq 5$, and that $B$ is a weight 3 block in a Scopes class $\mathcal{C}$ which forms exactly one [3:2]-pair with a Scopes class $\mathcal{D}$, and no [3:1]-pairs. If the decomposition numbers for blocks in $\mathcal{D}$ are all at most 1 and Theorem 1.1 holds over $\mathbb{F}$ with e replaced by $e-1$, then the decomposition numbers for $B$ are all at most 1 .

Proof. By Lemma 3.4, we may assume that $B$ is the block with the

$$
\left\langle 3,5,7, \ldots, 1+2 a,(3+2 a)^{c},(4+2 a)^{d}, 6+2 a, 8+2 a, \ldots, 4+2 a+2 b\right\rangle
$$


notation. This abacus may be pictured as follows:

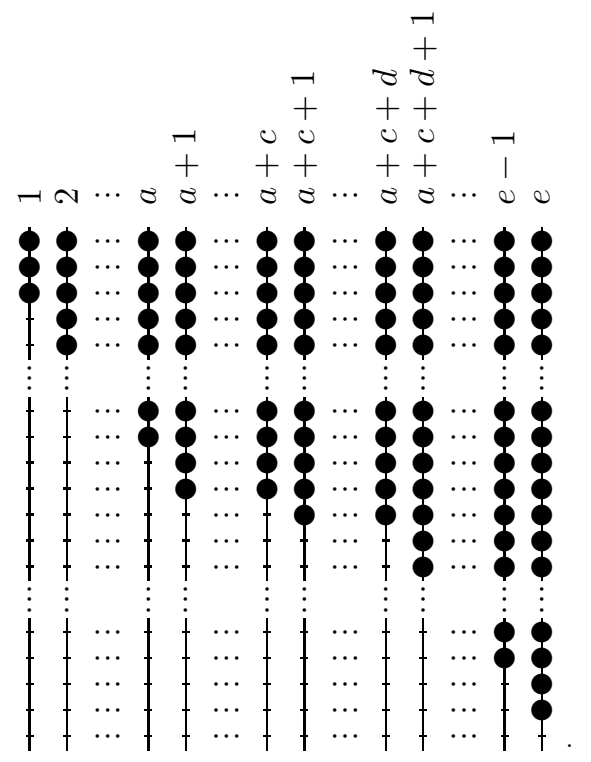

By replacing $B$ with its conjugate if necessary, we assume that $a \geq b$.

As in the proof of Proposition 3.7, we know that the decomposition number $\left[S^{\lambda}: D^{\mu}\right]$ for $B$ is at most 1 except possibly when $\lambda$ is one of the four exceptional partitions

$$
\begin{array}{ll}
\bar{\alpha}=[a+1], & \bar{\beta}=[a+1, a+c+d], \\
\bar{\gamma}=[a+1, a+c+d, a+c+d], & \bar{\delta}=[a+c+d, a+c+d, a+c+d] .
\end{array}
$$

So we assume that $\lambda$ is one of these four partitions. Assuming $\left[S^{\lambda}: D^{\mu}\right]>1$, we have $\left[S^{\bar{\alpha}}: D^{\mu}\right],\left[S^{\bar{\beta}}: D^{\mu}\right],\left[S^{\bar{\gamma}}: D^{\mu}\right],\left[S^{\bar{\delta}}: D^{\mu}\right] \geq 1$ by Proposition $2.10(4)$, so that

$$
\mu \unrhd \bar{\alpha}, \bar{\beta}, \bar{\gamma}, \bar{\delta} \unrhd \mu^{\diamond \prime}
$$

If $a \geq 1$, then $\lambda$ and $\mu$ may both be displayed on an abacus with an empty runner, namely the same abacus as above with three fewer beads on each runner. We define $\lambda^{-}$and $\mu^{-}$to be the partitions obtained by removing this runner, as in Section 1.1.8. Then if $\mu^{-}$is $(e-1)$-regular, we may equate $\left[S^{\lambda}: D^{\mu}\right]$ with a decomposition number for a Hecke algebra at an $(e-1)$ th root of unity; by our inductive assumption, this decomposition number is at most 1.

So we assume from now on that either $a=0$ or $\mu^{-}$is $(e-1)$-singular. If $a \geq 2$, then (assuming $\mu \unrhd \bar{\alpha}) \mu^{-}$is always $(e-1)$-regular, so we are left with only the cases where $a \leq 1$.

$[a=1 \geq b]$ : If we assume that $\mu \unrhd \bar{\alpha}, \mu^{-}$is $(e-1)$-singular and $\mu$ is not lowerable, then we find that $\mu$ is one of the following partitions:

$$
\begin{array}{ll}
{[c+2,2]} & (b=0 \text { or } 1), \\
{[e, 2],[e, e, 2],[e, c+2,2]} & (b=1), \\
{[2, e]} & (b=c=1) .
\end{array}
$$


Each of these partitions satisfies

$$
\begin{gathered}
\mu_{1}^{\prime}=2 e-2+d+3 b, \\
\mu_{2}^{\prime}=\mu_{3}^{\prime}=e-1+d+3 b .
\end{gathered}
$$

But we also have

$$
\bar{\alpha}_{1}^{\prime}=2 e-2+d+3 b, \quad \bar{\alpha}_{2}^{\prime}=\bar{\alpha}_{3}^{\prime}=e-1+d+3 b
$$

and similarly for $\bar{\beta}$ and $\bar{\gamma}$, and so we may apply Theorem 1.11: we define

$$
\bar{\mu}=\left(\mu_{1}-3, \mu_{2}-3, \ldots, \mu_{e-1+d+3 b}-3\right),
$$

and $\overline{\bar{\alpha}}, \overline{\bar{\beta}}, \overline{\bar{\gamma}}$ similarly. Then by Theorem $1.11(2)$ we have

$$
\begin{aligned}
& {\left[S^{\bar{\alpha}}: D^{\mu}\right]=\left[S^{\overline{\bar{\alpha}}}: D^{\bar{\mu}}\right],} \\
& {\left[S^{\bar{\beta}}: D^{\mu}\right]=\left[S^{\overline{\bar{\beta}}}: D^{\bar{\mu}}\right],} \\
& {\left[S^{\bar{\gamma}}: D^{\mu}\right]=\left[S^{\bar{\gamma}}: D^{\bar{\mu}}\right] .}
\end{aligned}
$$

By assumption, the decomposition numbers on the left are all positive. On the other hand, the decomposition numbers on the right are decomposition numbers for a block of weight 2 , and so are at most 1 . So all of these decomposition numbers equal 1.

Examining the weight 2 block in which $\bar{\mu}$ lies, we have

$$
\begin{aligned}
& \overline{\bar{\alpha}}=[c+1], \\
& \overline{\bar{\beta}}=[c+1, c+d+1], \\
& \overline{\bar{\gamma}}=[c+d+1, c+d+1]
\end{aligned}
$$

in the $\left\langle 5,6,5^{c-1}, 6^{d}, 8^{b}\right\rangle$ notation for partitions of weight 2 . So we may calculate

$$
\partial \overline{\bar{\alpha}}=d, \quad \partial \overline{\bar{\beta}}=d-1, \quad \partial \overline{\bar{\gamma}}=d .
$$

Hence by Corollary 2.2 we must have $\bar{\mu}=\overline{\bar{\alpha}}$, whence $\mu=\bar{\alpha}$. But then $\left[S^{\lambda}: D^{\mu}\right] \leq 1$ by Proposition $2.10(2)$.

$[a=0]$ : We examine Table 1 (putting $x=c, y=0, z=d$ ) to find those partitions $\mu$ such that $\mu \unrhd \bar{\alpha}$ and $\mu$ is not lowerable. These correspond to the cases $A_{E}, A_{G}, A_{J}, C_{E}, C_{G}$, and in none of these cases do we have $\bar{\delta} \unrhd \mu^{\diamond \prime}$.

3.4. Theorem 1.1 holds when $\operatorname{char}(\mathbb{F})=\infty$. We can now prove Theorem 1.1 for fields of infinite characteristic by induction on $e$. The cases $e=2,3,4$ can be dealt with using the LLT algorithm [11], so we suppose that $e \geq 5$. Let $\mathcal{C}$ denote the Scopes class in which $B$ lies, and assume that the result is true for blocks in all classes $\mathcal{D}$ with $\mathcal{D} \succ \mathcal{C}$.

If there are Scopes classes $\mathcal{D}_{1}, \mathcal{D}_{2}$ satisfying the conditions of Lemma 3.3, then by the conclusion of that result and by our assumption on the decomposition numbers for $\mathcal{D}_{1}, \mathcal{D}_{2}$ we find that the result holds. So we assume that the hypotheses of Lemma 3.3 do not hold, so that we are in one of cases $(\mathrm{C} 1)-(\mathrm{C} 4)$. Case C1 is dealt with by Theorem 2.11, case C2 by Proposition 3.5, case C3 by Proposition 3.7 and case C4 by Proposition 3.8. 


\section{Adjustment matrices in finite Characteristic}

In this section, we use the result of the previous section to prove Theorem 1.1 in general, by finding the adjustment matrices (as defined in Section 1.1.9) for weight 3 blocks. Given a weight 3 block $B$ of $\mathcal{H}_{n}$ over a field of finite characteristic and $e$-regular partitions $\lambda, \mu$ in $B$, let $B_{\lambda \mu}$ be the $(\lambda, \mu)$ entry of the adjustment matrix for $B$. Then we must prove the following theorem; throughout this section we employ the Kronecker delta.

Theorem 4.1 (James's Conjecture for weight 3 blocks). Suppose $\operatorname{char}(\mathbb{F}) \geq 5$, and that $B$ is a block of $\mathcal{H}_{n}$ of weight 3. Suppose that $\lambda$ and $\mu$ are e-regular partitions in $B$. Then $B_{\lambda \mu}=\delta_{\lambda \mu}$.

In this section, we assume that $e \geq 5$. Theorem 4.1 is true for $e=2,3,4$ and may be proved using exactly the same techniques, but there are various extra cases which are peculiar to these small values of $e$, and in the interests of brevity we do not consider these. In any case, to prove Theorem 1.1 in the symmetric group case, we need only consider $e \geq 5$.

First we note a trivial lemma which applies to adjustment matrices for blocks of any weight.

Lemma 4.2. Suppose $B$ is a block of $\mathcal{H}_{n}$ containing e-regular partitions $\lambda$ and $\mu$, and let $B^{\sharp}$ be the block conjugate to $B$. Then:

(1) $B_{\lambda \mu}=B_{\lambda^{\diamond} \mu^{\diamond}}^{\sharp}$;

(2) $B_{\lambda \mu}=0$ unless $\mu \triangleright \lambda$ and $\lambda^{\diamond \prime} \triangleright \mu^{\diamond \prime}$.

Proof.

(1) This follows easily from Corollary 1.5.

(2) If $B_{\lambda \mu}>0$, then $\left[S^{\nu}: D^{\mu}\right] \geq\left[S^{\nu}: D^{\lambda}\right]$ for all partitions $\nu$ in $B$. In particular, $\left[S^{\lambda}: D^{\mu}\right] \geq 1$, so that $\mu \triangleright \lambda$. Replacing $\lambda$ and $\mu$ with $\lambda^{\diamond}$ and $\mu^{\diamond}$ and applying part (1), we get $\lambda^{{ }^{\prime}} \triangleright \mu^{\diamond \prime}$.

In order to prove Theorem 4.1, we examine how the adjustment matrices of two blocks forming a $[3: \kappa]$-pair are related. Suppose $A$ and $B$ form a $[3: \kappa]$-pair with $A \prec B$; if $\kappa \leq 2$, recall from Sections 2.4 and 2.5 the exceptional simple modules in $A$ and $B$ for this pair. Recall also the map $\Phi$ defined in those sections or in $\$ 1.1 .5$.

Lemma 4.3. Suppose that $A$ and $B$ are as above. Suppose $\lambda, \mu$ are e-regular partitions in $B$.

(1) If $D^{\mu}$ is exceptional, then

$$
B_{\lambda \mu}=A_{\Phi(\lambda) \Phi(\mu)}=\delta_{\lambda \mu} .
$$

(2) If $D^{\lambda}$ is non-exceptional, then

$$
B_{\lambda \mu}=A_{\Phi(\lambda) \Phi(\mu)} .
$$

Proof.

(1) Since $D^{\mu}$ is exceptional, we must have $\kappa \leq 2$. But then we know all decomposition numbers $\left[S^{\nu}: D^{\mu}\right]$ by Corollary 2.6 and Proposition 2.10(2), and the result follows. 
(2) Let $\nu$ and $\xi$ be the $e$-cores of $A$ and $B$ respectively, and let $A^{0}$ and $B^{0}$ be the blocks of the algebras $\mathcal{H}_{n-\kappa}$ and $\mathcal{H}_{n}$ defined over $\mathbb{C}$ with $e$-cores $\nu$ and $\xi$. Suppose that $A^{0}$ and $B^{0}$ have decomposition matrices $\mathrm{C}$ and $\mathrm{D}$ respectively. Let $\mathrm{A}$ and $\mathrm{B}$ be the adjustment matrices for $A$ and $B$, so that $\mathrm{CA}$ and $\mathrm{DB}$ are the decomposition matrices for $A$ and $B$.

Let $\mathrm{S}$ be the 'Specht branching matrix', i.e. the matrix with rows indexed by partitions in $B$ and columns by partitions in $A$, in which the $(\lambda, \nu)$ entry is 1 if the abacus display for $\nu$ can be obtained from that for $\lambda$ by moving $\kappa$ beads one place to the left, and 0 otherwise. In other words, $\mathrm{S}$ has entries $\mathrm{S}_{\lambda \nu}$ such that $S^{\lambda} \downarrow_{A}^{B} \sim \sum_{\nu} \mathrm{S}_{\lambda \nu}\left(S^{\nu}\right)^{\kappa !}$ and $S^{\lambda} \downarrow_{A^{0}}^{B^{0}} \sim \sum_{\nu} \mathrm{S}_{\lambda \nu}\left(S^{\nu}\right)^{\kappa !}$. Let $\mathrm{T}^{0}$ be the 'simple branching matrix' for $B^{0}$ and $A^{0}$, i.e. the matrix with rows indexed by $e$-regular partitions in $B^{0}$ and columns indexed by $e$-regular partitions in $A^{0}$ in which the $(\lambda, \xi)$ entry is the composition multiplicity of $D^{\xi}$ in $D^{\lambda} \downarrow_{A^{0}}^{B^{0}}$. Let $\mathrm{T}$ be the simple branching matrix for $B$ and $A$, defined similarly.

Since restriction is an exact functor, we have

$$
\mathrm{DT}^{0}=\mathrm{SC}, \quad \mathrm{DBT}=\mathrm{SCA} .
$$

Hence $\mathrm{DBT}=\mathrm{DT}^{0} \mathrm{~A}$; since $\mathrm{D}$ has full column rank, we get

$$
\mathrm{BT}=\mathrm{T}^{0} \mathrm{~A} \text {. }
$$

(This is simply saying that restriction commutes with modular reduction.) Now if $D^{\mu}$ is a non-exceptional simple module in $B$, then we have

$$
\mathrm{T}_{\mu \Phi(\nu)}=\kappa ! \delta_{\mu \nu}
$$

by Theorem 1.3; on the other hand, if $D^{\mu}$ is an exceptional simple module in $B$, then we have $\mathrm{B}_{\lambda \mu}=\delta_{\lambda \mu}$ from (1). Hence we have

$$
(\mathrm{BT})_{\lambda \Phi(\nu)}=\kappa ! \mathrm{B}_{\lambda \Phi(\nu)}
$$

if $D^{\lambda}$ is non-exceptional. If $D^{\lambda}$ is a non-exceptional simple module in $B^{0}$, then we have

by Theorem 1.3 , and so

$$
\mathrm{T}_{\lambda \Phi(\mu)}^{0}=\kappa ! \delta_{\lambda \mu}
$$

$$
\left(\mathrm{T}^{0} \mathrm{~A}\right)_{\lambda \Phi(\nu)}=\kappa ! \mathrm{A}_{\lambda \Phi(\nu)}
$$

when $D^{\lambda}$ is non-exceptional. The result follows.

The following lemma will also be very useful; this is taken from the author's forthcoming paper [5] with Kai Meng Tan.

Lemma 4.4. Suppose $B$ is a weight 3 block of $\mathcal{H}_{n}$, and that $\operatorname{char}(\mathbb{F}) \geq 3$. If $\lambda$ and $\mu$ are e-regular partitions in $B$ with $\mu$ lowerable, then $B_{\lambda \mu}=\delta_{\lambda \mu}$.

Proof. Let $C$ be a block of $\mathcal{H}_{n-1}$ of weight 0,1 or 2 such that $D^{\mu} \downarrow_{C}^{B}=0$. Let $B^{0}$ and $C^{0}$ be the blocks of the algebras $\mathcal{H}_{n}$ and $\mathcal{H}_{n-1}$ defined over $\mathbb{C}$ corresponding to $B$ and $C$. Let $\mathrm{D}$ and $\mathrm{D}^{0}$ be the decomposition matrices for $B$ and $B^{0}$; our goal is to show that the columns of $\mathrm{D}$ and $\mathrm{D}^{0}$ corresponding to $\mu$ are equal. If we let $\mathrm{E}$ be the decomposition matrix for $C$, then by Theorem 2.1 (if $C$ has weight 2 ) or the discussion preceding it (if $C$ has weight 0 or 1 ) $\mathrm{E}$ is also the decomposition matrix of $C^{0}$. 
Let $\mathrm{S}$ be the 'Specht branching matrix' for restriction from $B$ to $C$, defined in the same way as in the proof of Lemma 4.3. Since the Branching Rule is independent of the characteristic, $\mathrm{S}$ is also the Specht branching matrix for restriction from $B^{0}$ to $C^{0}$. Let $\mathrm{T}$ be the 'simple branching matrix' for restriction from $B$ to $C$. By Theorem 1.3 the restriction of a simple module from $B$ to $C$ (or from $B^{0}$ to $C^{0}$ ) is either simple or zero, and if it is non-zero, it is described in a characteristic-free way, so $\mathrm{T}$ is also the simple branching matrix for restriction from $B^{0}$ to $C^{0}$.

By exactness of restriction from $B$ to $C$, we get

$$
\mathrm{DT}=\mathrm{SE},
$$

and by exactness of restriction from $B^{0}$ to $C^{0}$, we get

$$
\mathrm{D}^{0} \mathrm{~T}=\mathrm{SE},
$$

so that

$$
\mathrm{DT}=\mathrm{D}^{0} \mathrm{~T} .
$$

Let $\nu$ be the partition in $B$ such that $D^{\mu} \downarrow_{C}^{B} \cong D^{\nu}$. Then it is easy to see from Theorem 1.3 that $D^{\mu}$ is the only simple module in $B$ which restricts to give $D^{\nu}$. So the column of $\mathrm{T}$ corresponding to $\nu$ has a 1 in the $\mu$ position, and 0 s elsewhere. So the column of DT corresponding to $\nu$ equals the column of D corresponding to $\mu$, and the column of $\mathrm{D}^{0} \mathrm{~T}$ corresponding to $\nu$ equals the column of $\mathrm{D}^{0}$ corresponding to $\mu$. The result follows.

We shall use these results to prove that the adjustment matrices for all weight 3 blocks are trivial; for each $e$-regular partition, we induce or restrict the corresponding simple module until it becomes lowerable or it lies in a block whose decomposition matrix we know. To aid us, we introduce some notation for induction. Suppose $\mu$ is an $e$-regular partition lying in a block $B$, and take an abacus display for $B$. Suppose the number of beads on runner $i$ of the abacus exceeds the number of beads on the runner to the immediate right by $\kappa \geq 1$, and let $B_{i}$ be the block whose abacus is obtained by interchanging runner $i$ with the runner to its right. Then $B$ and $B_{i}$ form a $[3: \kappa]$-pair. If $D^{\mu}$ is a simple module in $B$ which is non-exceptional for this $[3: \kappa]$-pair, then define $\mathfrak{f}_{i}(\mu)$ to be the $e$-regular partition such that $D^{\mu} \uparrow_{B}^{B_{i}} \cong\left(D^{\mathfrak{f}_{i}(\mu)}\right)^{\oplus \kappa !}$, and leave $\mathfrak{f}_{i}(\mu)$ undefined otherwise (so if $\mathfrak{f}_{i}(\mu)$ is defined, then $\Phi\left(\mathfrak{f}_{i}(\mu)\right)=\mu$, where $\Phi$ is the map defined in 1.1.5, 2.4 or 2.5). We shall make use of the following two ideas.

(1) Suppose $\lambda$ is an $e$-regular partition lying in a block $B$, and that we can find $i_{1}, \ldots, i_{r}$ such that $\mathfrak{f}_{i_{r}} \ldots \mathfrak{f}_{i_{1}}(\lambda)$ is defined and lies in a block with trivial adjustment matrix. Then we have $B_{\lambda \mu}=\delta_{\lambda \mu}$ for all $e$-regular partitions $\mu$ in $B$, by Lemma $4.3(2)$.

(2) Suppose $\lambda$ and $\mu$ are $e$-regular partitions lying in a block $B$, and that we can find $i_{1}, \ldots, i_{r}$ such that $\mathfrak{f}_{i_{r}} \ldots \mathfrak{f}_{i_{1}}(\lambda)$ and $\mathfrak{f}_{i_{r}} \ldots \mathfrak{f}_{i_{1}}(\mu)$ are defined, with the latter partition being lowerable. Then $B_{\lambda \mu}=\delta_{\lambda \mu}$, by Lemma $4.3(2)$ and Lemma 4.4 .

Our strategy will be to use induction on $n$ (in the usual direction), but we shall also make use of the fact [8, Corollary 4] that the adjustment matrix for a Rouquier block is trivial.

To begin with, we examine the block of $\mathcal{H}_{3 e}$ with core (0). As noted in the proof of Proposition 3.5, every e-regular partition in this block is lowerable. So the adjustment matrix of this block is trivial, by Lemma 4.4. So we may assume that 
$B$ has a non-empty core, and so there is at least one block $A$ such that $A$ and $B$ form a $[3: \kappa]$-pair. Suppose $\lambda$ and $\mu$ are $e$-regular partitions in $B$. If either $D^{\mu}$ is

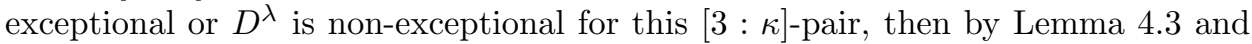
by induction we have $B_{\lambda \mu}=\delta_{\lambda \mu}$. So we may assume that $D^{\lambda}$ is exceptional and $D^{\mu}$ is non-exceptional for every such $[3: \kappa]$-pair. If there are two blocks $A$ and $A^{\prime}$ forming $[3: \kappa]$-pairs with $B$, then there is no $\lambda$ such that $D^{\lambda}$ is exceptional for both $[3: \kappa]$-pairs. So it suffices to consider only those cases where there is exactly one block forming a $[3: \kappa]$-pair with $B$, and where $\kappa \leq 2$.

4.1. Blocks with rectangular cores. The aim of this subsection is to prove the following.

Proposition 4.5. Suppose $\operatorname{char}(\mathbb{F}) \geq 5$ and $B$ is a weight 3 block of $\mathcal{H}_{n}$, and that there is exactly one block $A$ forming a $[3: \kappa]$-pair with $B$, and $\kappa=1$. If the adjustment matrix for $A$ is trivial, then the adjustment matrix for $B$ is trivial.

If $B$ satisfies the hypotheses of Proposition 4.5, then the core of $B$ has the form $x^{z}$ for some $x$ and $z$ with $x+z \leq e$. By Lemma 4.3(2), we may assume that $\lambda=\alpha_{i}$ for some $i$ (that is, $\lambda=[x+y+1, x+y+1]$ or $[x+y+1, x+y+1, k]$ for some $k \neq x$ ), and by Lemma 4.4, we may assume that $\mu$ is not lowerable, so that $\mu$ is one of the partitions listed in Table 1 . If we let $\pi$ be the permutation described in Section 2.4 and $\lambda=\alpha_{\pi(j)}$, then by Lemma 4.2(2) we may assume that $\mu \triangleright \alpha_{\pi(j)}$ and $\gamma_{\pi(j+1)} \triangleright \mu^{\diamond \prime}$. Looking at Table 1, we find that in the cases $\mathrm{A}_{\mathrm{J}}, \mathrm{A}_{\mathrm{K}}, \mathrm{A}_{\mathrm{L}}, \mathrm{A}_{\mathrm{M}}$, $A_{N}, C_{L}, C_{M}, C_{N}, G_{M}, J_{A}, K_{A}, L_{A}, L_{C}, M_{A}, M_{C}, M_{G}, N_{A}, N_{C}$ there is no such $j$. So we may assume that $\mu$ is not one of these partitions.

4.1.1. Inducing $D^{\lambda}$ and $D^{\mu}$. Consider the (partial) function $\mathfrak{f}=\mathfrak{f}_{x+y+1} \mathfrak{f}_{x+y+2} \ldots \mathfrak{f}_{e}$. The effect of this is to move each of the runners $e, e-1, \ldots, x+y+1$ in succession past runner $x+1$ (if $y>0$ ) or runner 1 (if $y=0$ ). It is easy to see that $\mathfrak{f}(\lambda)$ is defined and that if $\mu$ is one of

$$
\begin{gathered}
{[x+1, x+y+1](\text { with } y \geq 1, z=1), \quad[1, x+y+1, x+1](\text { with } y \geq 1),} \\
{[x+y+1, x+y+2, x+1](\text { with } y \geq 1),} \\
{[x+y+1, x+1, x+2](\text { with } y \geq 1), \quad[1, x+y+1](\text { with } y=0),}
\end{gathered}
$$

then $\mathfrak{f}(\mu)$ is defined and lowerable. For example, $\mathfrak{f}([1, x+1, x+y+1])$ is the partition $\left[x+1,1 \mid 3^{x+1}, 4^{z}, 3^{y-1}\right]$; we easily see that this is lowerable from its abacus display:

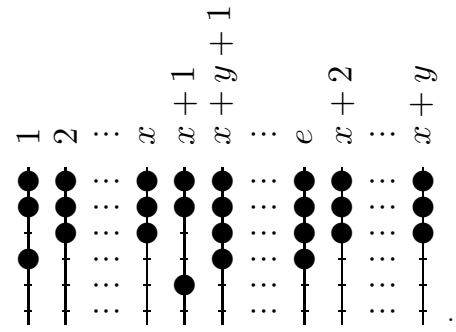

So we find that in the cases $\mathrm{B}_{\mathrm{G}}, \mathrm{B}_{\mathrm{H}}, \mathrm{B}_{\mathrm{I}}, \mathrm{E}_{\mathrm{A}}, \mathrm{E}_{\mathrm{C}}, \mathrm{F}_{\mathrm{D}}, \mathrm{H}_{\mathrm{A}}, \mathrm{H}_{\mathrm{B}}, \mathrm{H}_{\mathrm{C}}, \mathrm{H}_{\mathrm{G}}, \mathrm{H}_{\mathrm{H}}, \mathrm{H}_{\mathrm{I}}, \mathrm{I}_{\mathrm{A}}$, $\mathrm{I}_{\mathrm{B}}, \mathrm{I}_{\mathrm{C}}, \mathrm{I}_{\mathrm{G}}, \mathrm{I}_{\mathrm{H}}$ we have $B_{\lambda \mu}=\delta_{\lambda \mu}$. Applying the Mullineux map and using Lemma 4.2(1), we may also deal with the cases $A_{E}, A_{H}, A_{I}, C_{E}, C_{H}, C_{I}, D_{F}, G_{B}, G_{H}, G_{I}$. 
4.1.2. The case $\mu=[x+1, x+2, x+3]$. Now assume that $y=0$ and $z \geq 3$ and suppose $\mu=[x+1, x+2, x+3]$. By Lemma 4.2(2), we may assume that $\lambda$ is one of the exceptional partitions

$$
[x+1, x+1, x+3], \quad[x+1, x+1, x+2], \quad[x-1, x+1, x+1](\text { if } x \geq 2) .
$$

First we look at $\lambda=[x+1, x+1, x+3]$. We apply the partial function $\mathfrak{f}=$ $\left(\mathfrak{f}_{x+3} \mathfrak{f}_{x+4} \cdots \mathfrak{f}_{e}\right)^{x+2}$ to both $\lambda$ and $\mu$. To make it easier to see what is happening, we apply this in stages: for $\mu$, it is easy to see that

$$
\left(\mathfrak{f}_{x+3} \ldots \mathfrak{f}_{e}\right)^{x}(\mu)=\left[x+1, x+2, x+3 \mid 3^{x}, 5^{z-2}, 4^{2}\right],
$$

with abacus display

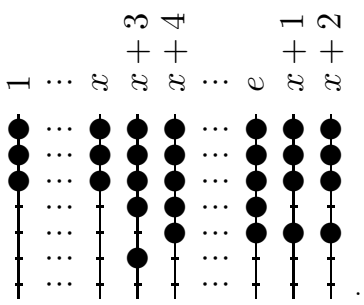

Applying $\left(\mathfrak{f}_{x+3} \ldots \mathfrak{f}_{e}\right)$ again, we reach $\left[x+1, x+2 \mid 3^{x}, 4,5^{z-2}, 4\right]$ :

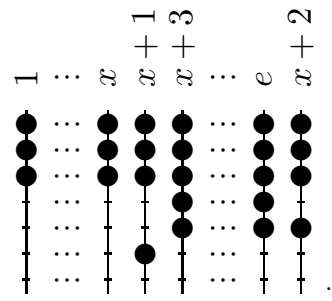

Applying $\left(\mathfrak{f}_{x+3} \ldots \mathfrak{f}_{e}\right)$ once more, we find $\mathfrak{f}(\mu)=\left[x+1, x+2 \mid 3^{x}, 4^{2}, 5^{z-2}\right]$. For $\lambda$, applying $\left(\mathfrak{f}_{x+3} \ldots \mathfrak{f}_{e}\right)^{x}$ yields $\left[x+1, x+1, x+3 \mid 3^{x}, 5^{z-2}, 4^{2}\right]$ :

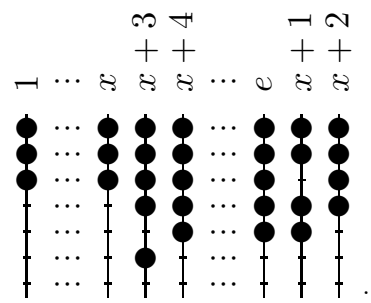

Applying $\left(\mathfrak{f}_{x+3} \ldots \mathfrak{f}_{e}\right)$ again yields $\left[x+1, x+1 \mid 3^{x}, 4,5^{z-2}, 4\right]$ :

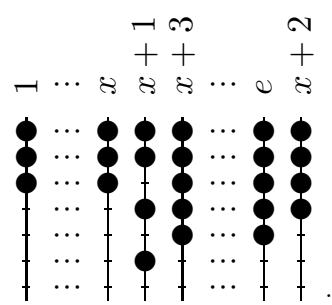


We apply $\left(\mathfrak{f}_{x+3} \ldots \mathfrak{f}_{e}\right)$ once more to find $\mathfrak{f}(\lambda)=\left[x+1, x+1 \mid 3^{x}, 4^{2}, 5^{z-2}\right]$. Let $\hat{B}$ be the block in which $\mathfrak{f}(\lambda)$ and $\mathfrak{f}(\mu)$ lie.

A very simple application of the Jantzen-Schaper formula yields $\left[S^{\mathfrak{f}(\lambda)}: D^{\mathfrak{f}(\mu)}\right]=$ 1 , irrespective of the underlying characteristic, which means that $\hat{B}_{\mathfrak{f}(\lambda) \mathfrak{f}(\mu)}=0$, and so $B_{\lambda \mu}=0$ by Lemma 4.3 .

Next we look at $\lambda=[x+1, x+1, x+2]$. Here we find a sequence $i_{1}, \ldots, i_{r}$ such that $\mathfrak{f}_{i_{1}} \ldots \mathfrak{f}_{i_{r}}(\lambda)$ is defined and lies in a Rouquier block; by Lemma 4.3(2) and [8, Corollary 4] we shall have $B_{\lambda \mu}=0$. Again, we apply the functions $\mathfrak{f}_{i}$ in stages.

We have $\lambda=\left[x+1, x+1, x+2 \mid 3^{x}, 4^{z}\right]$; we apply $\mathfrak{f}_{e}^{2 e-2}$ followed by $\left(\mathfrak{f}_{e-1} \mathfrak{f}_{e}\right)^{2 e-4}$ and then $\left(\mathfrak{f}_{e-2} \mathfrak{f}_{e-1} \mathfrak{f}_{e}\right)^{2 e-6}$ and so on in succession up to $\left(\mathfrak{f}_{x+3} \ldots \mathfrak{f}_{e}\right)^{2 x+4}$, and we reach the partition $\left[x+1, x+1, x+2 \mid 3^{x}, 4^{2}, 6,8, \ldots, 2 z\right]$ :

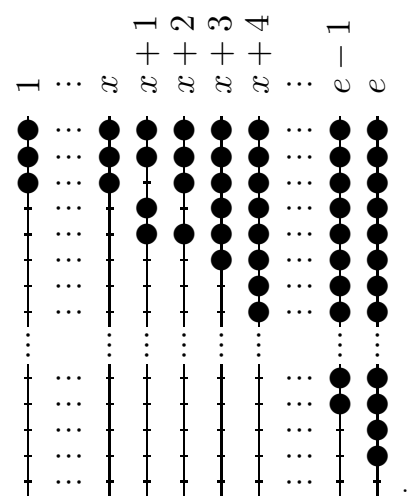

We apply $\left(\mathfrak{f}_{x+2} \ldots \mathfrak{f}_{e}\right)^{x+1}$ to reach $\left[x+1, x+1 \mid 3^{x}, 4,5,7,9, \ldots, 2 z+1\right]$ :

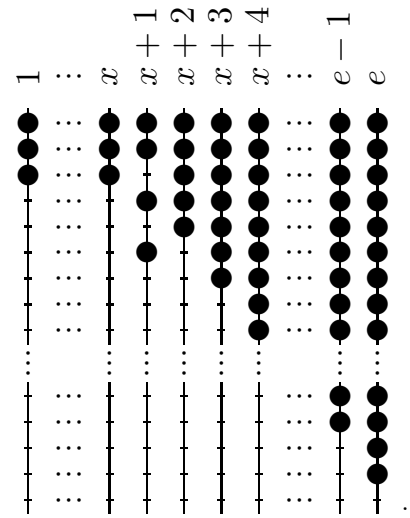

Now we apply $\left(\mathfrak{f}_{x+2} \ldots \mathfrak{f}_{e}\right)^{x+1}$ again followed by $\left(\mathfrak{f}_{x+1} \ldots \mathfrak{f}_{e}\right)^{x}$ to reach $[x+1, x+1 \mid$ $\left.3^{x}, 5,7, \ldots, 2 z+3\right]$, and then we apply $\left(\mathfrak{f}_{x} \ldots \mathfrak{f}_{e}\right)^{2 x-2}$ and then $\left(\mathfrak{f}_{x-1} \ldots \mathfrak{f}_{e}\right)^{2 x-4}$ and 
so on up to $\left(\mathfrak{f}_{2} \ldots \mathfrak{f}_{e}\right)^{2}$ to reach $[x+1, x+1 \mid 3,5,7, \ldots, 2 e+1]$, which lies in a Rouquier block.

Finally, we suppose that $x \geq 2$ and consider $\lambda=[x-1, x+1, x+1]$. We apply the Mullineux map to $\lambda$ and $\mu$ to get

$$
\begin{aligned}
& \mu^{\diamond}= \begin{cases}{\left[z+1, z+2, z+3 \mid 3^{z}, 4^{x}\right]} & (x \geq 3), \\
{\left[z+1, z+2 \mid 3^{z}, 4^{x}\right]} & (x=2),\end{cases} \\
& \lambda^{\diamond}= \begin{cases}{\left[z+1, z+1, z+3 \mid 3^{z}, 4^{x}\right]} & (x \geq 3), \\
{\left[z+1, z+1 \mid 3^{z}, 4^{x}\right]} & (x=2) .\end{cases}
\end{aligned}
$$

The case $x \geq 3$ corresponds to a case we have already considered. In the case $x=2$, a simple application of the Jantzen-Schaper formula yields $\left[S^{\lambda^{\diamond}}: D^{\mu^{\diamond}}\right]=1$ regardless of the underlying characteristic. So we have $B_{\lambda^{\diamond} \mu^{\diamond}}^{\sharp}=0$, and so $B_{\lambda_{\mu}}=0$.

Hence we have $B_{\lambda \mu}=\delta_{\lambda \mu}$ in the cases $\mathrm{G}_{\mathrm{A}}, \mathrm{G}_{\mathrm{C}}$ and $\mathrm{G}_{\mathrm{G}}$. By looking at conjugate cases we also deal with the cases $\mathrm{A}_{\mathrm{G}}$ and $\mathrm{C}_{\mathrm{G}}$, and so Proposition 4.5 is proved.

\subsection{Blocks with birectangular cores.}

Proposition 4.6. Suppose that $\operatorname{char}(\mathbb{F}) \geq 5$, that $B$ is a weight 3 block of $\mathcal{H}_{n}$, and that there is exactly one block $A$ forming a $[3: \kappa]$-pair with $B$, with $\kappa=2$. If the adjustment matrix for $A$ is trivial, then the adjustment matrix for $B$ is trivial.

If $B$ is a weight 3 block as in Proposition 4.6, then $B$ has a core of the form $\left((w+x)^{z}, w^{y+z}\right)$ for some $w, x, y, z \geq 0$ with $w+x+y+z=e$ and $w, z>0$. This may be represented on an abacus with the $\left\langle 3^{w}, 5^{z}, 4^{y}, 3^{x}\right\rangle$ notation. By Lemma 4.3(2) we have $B_{\lambda \mu}=\delta_{\lambda \mu}$ for $e$-regular partitions $\lambda, \mu$ in $B$, except possibly when $D^{\lambda}$ is the unique exceptional simple module, i.e. when $\lambda=[w+x+y+1, w+x+$ $\left.y+1, w+x+y+1 \mid 3^{w}, 5^{z}, 4^{y}, 3^{x}\right]$. As with the partition $[x+1, x+1, x+2]$ in the last section, we shall induce this simple module up to a Rouquier block. The behaviour varies depending on whether $x$ and $y$ are positive, so we must consider four cases. First suppose $x, y>0$. We first apply $\left(\mathfrak{f}_{w+x+y+2} \ldots \mathfrak{f}_{e}\right)^{x+y}$ to reach $\left[w+x+y+1, w+x+y+1, w+x+y+1 \mid 3^{w}, 5,4^{y}, 3^{x}, 5^{z-1}\right]:$

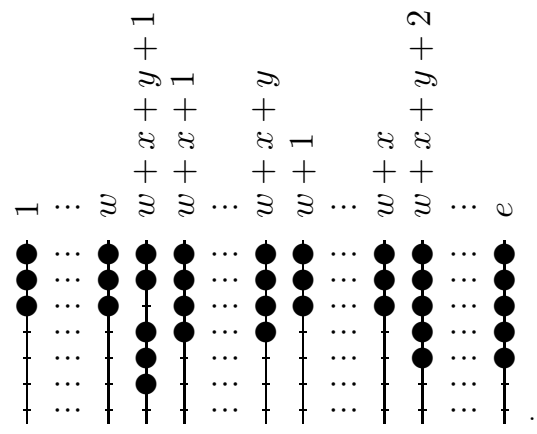


Next we apply $\mathfrak{f}_{w+x+y+1}{ }^{x+y}$ and reach $\left[w+x+1, w+x+1, w+x+y+1 \mid 3^{w}, 4^{y}, 3^{x}, 5^{z}\right]$ :

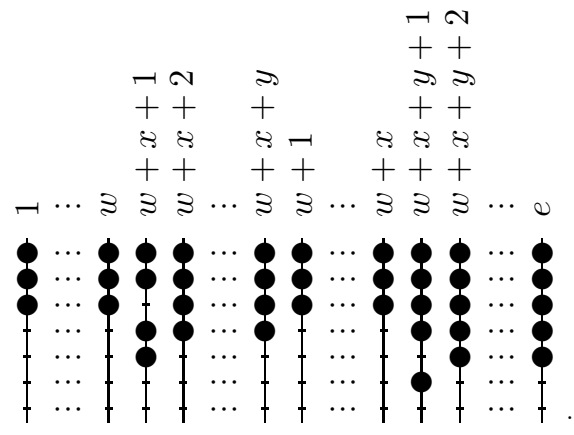

Applying $\left(\mathfrak{f}_{w+x+2} \ldots \mathfrak{f}_{w+x+y}\right)^{x}$ we reach $[w+x+1, w+x+1, w+x+y+1$ | $\left.3^{w}, 4,3^{x}, 4^{y-1}, 5^{z}\right]$. Next we apply $\mathfrak{f}_{w+x+1}^{x}$ and reach $[w+1, w+x+1, w+x+y+1 \mid$ $3^{w+x}, 4^{y}, 5^{z}$. Now we apply the following functions in turn:

$$
\begin{aligned}
& \mathfrak{f}_{e}^{2 e-2}, \quad\left(\mathfrak{f}_{e-1} \mathfrak{f}_{e}\right)^{2 e-4}, \quad \ldots, \quad\left(\mathfrak{f}_{w+x+y+2} \ldots \mathfrak{f}_{e}\right)^{2 w+2 x+2 y+2}, \\
& \left(\mathfrak{f}_{w+x+y+1} \ldots \mathfrak{f}_{e}\right)^{w+x+y}, \\
& \left(\mathfrak{f}_{w+x+y} \ldots \mathfrak{f}_{e}\right)^{2 w+2 x+2 y-2}, \quad \ldots, \quad\left(\mathfrak{f}_{w+x+2} \ldots \mathfrak{f}_{e}\right)^{2 w+2 x+2}, \\
& \left(\mathfrak{f}_{w+x+1} \ldots \mathfrak{f}_{e}\right)^{w+x}, \\
& \left(\mathfrak{f}_{w+x} \ldots \mathfrak{f}_{e}\right)^{2 w+2 x-2}, \quad \ldots, \quad\left(\mathfrak{f}_{2} \ldots \mathfrak{f}_{e}\right)^{2} .
\end{aligned}
$$

We reach $[w+1, w+x+1, w+x+y+1 \mid 3,5,7, \ldots, 2 e+1]$, which lies in a Rouquier block.

The other three cases are similar but simpler, and we give much less detail. If $x>y=0$, then we can find a sequence $i_{1}, \ldots, i_{r}$ such that $\mathfrak{f}_{i_{1}}, \ldots, \mathfrak{f}_{i_{r}}(\lambda)$ is defined and equals $[w+1, w+x+y+1, w+x+y+1 \mid 3,5,7, \ldots, 2 e+1]$. If $y>x=0$, then we can reach $[w+1, w+1, w+x+y+1 \mid 3,5,7, \ldots, 2 e+1]$, and if $x=y=0$, then we can reach $[w+x+y+1, w+x+y+1, w+x+y+1 \mid 3,5,7, \ldots, 2 e+1]$.

By Lemma 4.3(2) and since the adjustment matrix of a weight 3 Rouquier block is trivial, we have $B_{\lambda \mu}=\delta_{\lambda \mu}$ for all $\mu$. So Proposition 4.6 is proved, and this completes the proof of Theorem 1.1 by induction.

\section{Appendix A. Non-LOWERABlE PARTitions in BLOCKS WITH RECTANGULAR CORES}

Table 1 is a list of all $e$-regular partitions which are not lowerable in blocks with rectangular cores. See Section 2.7 for more details.

\section{Appendix B. Application of the Jantzen-Schaper formula}

In this section, we illustrate the explicit calculations carried out using the Jantzen-Schaper formula in the proof of Proposition 3.5. For the partitions $\mu=$ $[e, 2]$ and $[e, e, 2]$, we give a table of the coefficients $c_{\lambda \nu}$ for all $\mu \unrhd \lambda \triangleright \nu \unrhd \mu^{\diamond \prime}$. At the right we list the possible decomposition numbers $\left[S^{\lambda}: D^{\mu}\right]$ satisfying Theorem 1.6 and Proposition 1.7; the reader is invited to check that these are the only possibilities. 
TABle 1

\begin{tabular}{|c|c|c|c|}
\hline & $\mu$ & conditions on $x, y, z$ & $\mu^{\diamond \prime}$ \\
\hline $\mathrm{A}_{\mathrm{E}}$ & {$[x+y+1]$} & $y=0, z=2$ & {$[e, e, x]$} \\
\hline $\mathrm{A}_{\mathrm{G}}$ & {$[x+y+1]$} & $y=0, z=1$ & {$[x, x-1, x-2]$} \\
\hline$A_{H}$ & {$[x+y+1]$} & $y=1, z=1$ & {$[x+y, x, x-1]$} \\
\hline $\mathrm{A}_{\mathrm{I}}$ & {$[x+y+1]$} & $y=2, z=1$ & {$[x+y, x+y-1, x]$} \\
\hline $\mathrm{A}_{J}$ & {$[x+y+1]$} & $y=0, z \geq 3$ & {$[e, e, e-1]$} \\
\hline$A_{K}$ & {$[x+y+1]$} & $y \geq 1, z \geq 3$ & {$[e, e-1, x+y]$} \\
\hline$A_{L}$ & {$[x+y+1]$} & $y \geq 2, z=2$ & {$[e, x+y, x+y-1]$} \\
\hline$A_{M}$ & {$[x+y+1]$} & $y \geq 3, z=1$ & {$[x+y, x+y-1, x+y-2]$} \\
\hline$A_{N}$ & {$[x+y+1]$} & $y=1, z=2$ & {$[e, e, x+y]$} \\
\hline$\overline{\mathrm{B}_{\mathrm{G}}}$ & {$[x+1, x+y+1]$} & $y=1, z=1$ & {$[x, x-1, x-2]$} \\
\hline $\mathrm{B}_{\mathrm{H}}$ & {$[x+1, x+y+1]$} & $y=2, z=1$ & {$[x+y, x, x-1]$} \\
\hline $\mathrm{B}_{\mathrm{I}}$ & {$[x+1, x+y+1]$} & $y \geq 3, z=1$ & {$[x+y, x+y-1, x]$} \\
\hline $\mathrm{C}_{\mathrm{E}}$ & {$[x+y+1, x+y+2]$} & $y=0, z \geq 3$ & {$[e, e, x]$} \\
\hline $\mathrm{C}_{\mathrm{G}}$ & {$[x+y+1, x+y+2]$} & $y=0, z=2$ & {$[x, x-1, x-2]$} \\
\hline $\mathrm{C}_{\mathrm{H}}$ & {$[x+y+1, x+y+2]$} & $y=1, \quad z=2$ & {$[x+y, x, x-1]$} \\
\hline $\mathrm{C}_{\mathrm{I}}$ & {$[x+y+1, x+y+2]$} & $y=2, z=2$ & {$[x+y, x+y-1, x]$} \\
\hline $\mathrm{C}_{\mathrm{L}}$ & {$[x+y+1, x+y+2]$} & $y \geq 2, \quad z \geq 3$ & {$[e, x+y, x+y-1]$} \\
\hline $\mathrm{C}_{\mathrm{M}}$ & {$[x+y+1, x+y+2]$} & $y \geq 3, z=2$ & {$[x+y, x+y-1, x+y-2]$} \\
\hline $\mathrm{C}_{\mathrm{N}}$ & {$[x+y+1, x+y+2]$} & $y=1, z \geq 3$ & {$[e, e, x+y]$} \\
\hline$\overline{D_{F}}$ & {$[x+y+1, x+1]$} & $y \geq 1$ & {$[e, x+y, x]$} \\
\hline $\mathrm{E}_{\mathrm{A}}$ & {$[1, x+y+1]$} & $x=2, y=0$ & {$[x, x, x]$} \\
\hline $\mathrm{E}_{\mathrm{C}}$ & {$[1, x+y+1]$} & $x \geq 3, y=0$ & {$[x, x, x-1]$} \\
\hline $\mathrm{F}_{\mathrm{D}}$ & {$[1, x+y+1, x+1]$} & $y \geq 1$ & {$[x+y, x, x]$} \\
\hline $\mathrm{G}_{\mathrm{A}}$ & {$[x+y+1, x+y+2, x+y+3]$} & $x=1, y=0$ & {$[x, x, x]$} \\
\hline $\mathrm{G}_{\mathrm{B}}$ & {$[x+y+1, x+y+2, x+y+3]$} & $x=1, y=1$ & {$[x+y, x+y, x]$} \\
\hline $\mathrm{G}_{\mathrm{C}}$ & {$[x+y+1, x+y+2, x+y+3]$} & $x=2, y=0$ & {$[x, x, x-1]$} \\
\hline $\mathrm{G}_{\mathrm{G}}$ & {$[x+y+1, x+y+2, x+y+3]$} & $x \geq 3, y=0, z \geq 3$ & {$[x, x-1, x-2]$} \\
\hline $\mathrm{G}_{\mathrm{H}}$ & {$[x+y+1, x+y+2, x+y+3]$} & $x \geq 2, y=1, z \geq 3$ & {$[x+y, x, x-1]$} \\
\hline $\mathrm{G}_{\mathrm{I}}$ & {$[x+y+1, x+y+2, x+y+3]$} & $y=2, \quad z \geq 3$ & {$[x+y, x+y-1, x]$} \\
\hline $\mathrm{G}_{\mathrm{M}}$ & {$[x+y+1, x+y+2, x+y+3]$} & $y \geq 3, z \geq 3$ & {$[x+y, x+y-1, x+y-2]$} \\
\hline$\overline{\mathrm{H}_{\mathrm{A}}}$ & {$[x+y+1, x+y+2, x+1]$} & $x=1, y=1$ & {$[x, x, x]$} \\
\hline $\mathrm{H}_{\mathrm{B}}$ & {$[x+y+1, x+y+2, x+1]$} & $x=1, y=2$ & {$[x+y, x+y, x]$} \\
\hline $\mathrm{H}_{\mathrm{C}}$ & {$[x+y+1, x+y+2, x+1]$} & $x=2, y=1$ & {$[x, x, x-1]$} \\
\hline $\mathrm{H}_{\mathrm{G}}$ & {$[x+y+1, x+y+2, x+1]$} & $x \geq 3, y=1, z \geq 2$ & {$[x, x-1, x-2]$} \\
\hline $\mathrm{H}_{\mathrm{H}}$ & {$[x+y+1, x+y+2, x+1]$} & $x \geq 2, y=2, z \geq 2$ & {$[x+y, x, x-1]$} \\
\hline $\mathrm{H}_{\mathrm{I}}$ & {$[x+y+1, x+y+2, x+1]$} & $y \geq 3, z \geq 2$ & {$[x+y, x+y-1, x]$} \\
\hline $\mathrm{I}_{\mathrm{A}}$ & {$[x+y+1, x+1, x+2]$} & $x=1, y=2$ & {$[x, x, x]$} \\
\hline$I_{B}$ & {$[x+y+1, x+1, x+2]$} & $x=1, y \geq 3$ & {$[x+y, x+y, x]$} \\
\hline $\mathrm{I}_{\mathrm{C}}$ & {$[x+y+1, x+1, x+2]$} & $x=2, y=2$ & {$[x, x, x-1]$} \\
\hline$I_{G}$ & {$[x+y+1, x+1, x+2]$} & $x \geq 3, y=2$ & {$[x, x-1, x-2]$} \\
\hline $\mathrm{I}_{\mathrm{H}}$ & {$[x+y+1, x+1, x+2]$} & $x \geq 2, y \geq 3$ & {$[x+y, x, x-1]$} \\
\hline $\mathrm{J}_{\mathrm{A}}$ & {$[1,2]$} & $x \geq 3, y=0$ & {$[x, x, x]$} \\
\hline $\mathrm{K}_{\mathrm{A}}$ & {$[1,2, x+1]$} & $x \geq 3, y \geq 1$ & {$[x, x, x]$} \\
\hline $\mathrm{L}_{\mathrm{A}}$ & {$[1, x+1, x+2]$} & $x=2, y \geq 2$ & {$[x, x, x]$} \\
\hline $\mathrm{L}_{\mathrm{C}}$ & {$[1, x+1, x+2]$} & $x \geq 3, y \geq 2$ & {$[x, x, x-1]$} \\
\hline$\overline{\mathrm{M}_{\mathrm{A}}}$ & {$[x+1, x+2, x+3]$} & $x=1, y \geq 3$ & {$[x, x, x]$} \\
\hline $\mathrm{M}_{\mathrm{C}}$ & {$[x+1, x+2, x+3]$} & $x=2, y \geq 3$ & {$[x, x, x-1]$} \\
\hline $\mathrm{M}_{\mathrm{G}}$ & {$[x+1, x+2, x+3]$} & $x \geq 3, y \geq 3$ & {$[x, x-1, x-2]$} \\
\hline$\overline{\mathrm{N}_{\mathrm{A}}}$ & {$[1, x+1]$} & $x=2, y=1$ & {$[x, x, x]$} \\
\hline $\mathrm{N}_{\mathrm{C}}$ & {$[1, x+1]$} & $x \geq 3, y=1$ & {$[x, x, x-1]$} \\
\hline
\end{tabular}


B.1. $\mu=[e, 2], \quad \mu^{\diamond^{\prime}}=[e-1, e-2,1]$.

\begin{tabular}{|c|c|c|c|c|c|c|c|c|c|c|c|c|c|c|c|c|}
\hline & 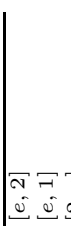 & & 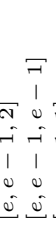 & $F$ & & $\begin{array}{cc}\pi \\
1 \\
1 \\
0 & 7 \\
i & 0 \\
1 & 1 \\
0 & 0 \\
0 & 0 \\
0 & 0\end{array}$ & & & & 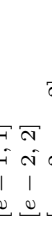 & 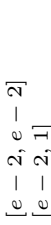 & 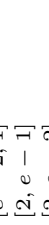 & & & 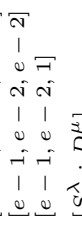 & 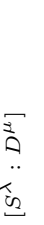 \\
\hline$[e, 2]$ & & & & & & & & & & . & & & & & & \\
\hline$[e, 1]$ & & & & & & & & . & & . & & & & . & & \\
\hline$[2, e]$ & 10 & & & & & & & & & . & & & & & . & \\
\hline$[e, e-1,2]$ & $\begin{array}{ll}-1 & 0\end{array}$ & & 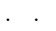 & & . & . & . & . & . & . & . & . & & . & . & 0 \\
\hline$[e, e-1, e$ & $\begin{array}{ll}0 & 0\end{array}$ & 1 & 1 . & & . & . & . & . & & . & . & . & & . & . & 1 \\
\hline$[e, e-1]$, & $\begin{array}{ll}0 & 1\end{array}$ & $0-$ & -11 & & & & & & & & & & & & & 1 \\
\hline$[e, e-2,2]$ & $10-$ & -1 & 10 & & & . & & & & & & . & & & & \\
\hline$[e, e-2, e-2]$ & $\begin{array}{ll}0 & 0-1\end{array}$ & $-1 \mathrm{c}-2 \cdot) \cdot$ & $\begin{array}{ll}0 & 1\end{array}$ & 0 & 1 & & & & & . & & . & & & . & \\
\hline$[e, e-2,1]$ & $0-1$ & 0 & $\begin{array}{ll}0 & 0 \\
-1\end{array}$ & $1-$ & -1 & & & & & & & . & & . & . & 0 \\
\hline & $\begin{array}{ll}-1 & 0\end{array}$ & 1 & $\begin{array}{ll}0 & 0\end{array}$ & & 0 & $\begin{array}{ll}0 & 0\end{array}$ & & & & . & & . & & & . & \\
\hline$[e-1,2$ & 10 & 0 & 10 & 0 & 0 & $\begin{array}{ll}0 & 0\end{array}$ & 1 & & & & & & & & & \\
\hline$[e-1, e-1]$ & $\begin{array}{ll}0 & 0\end{array}$ & 0 & $\begin{array}{ll}0 & 1\end{array}$ & 0 & 0 & 00 & 1 & 1 & & & & . & & & & \\
\hline$[e-$ & $0-1$ & 0 & $\begin{array}{ll}0 & 0\end{array}$ & 1 & 0 & 00 & & & & & & . & & & & \\
\hline$[e-2,2]$ & $\begin{array}{lll}-1 & 0\end{array}$ & 0 & $\begin{array}{ll}0 & 0\end{array}$ & 0 & 1 & $0 \quad 0$ & & 1 & 0 & & & . & & & & 0 \\
\hline$-2, e-2]$ & $\begin{array}{ll}0 & 0\end{array}$ & 0 & $\begin{array}{ll}0 & 0\end{array}$ & 0 & 0 & 10 & & & & & & & & & & \\
\hline & $\begin{array}{ll}0 & 1\end{array}$ & 0 & $\begin{array}{ll}0 & 0 \\
\end{array}$ & 0 & 0 & $0 \quad 1$ & 0 & 0 & 0 & $1-1$ & 1 & & & & & \\
\hline & $\begin{array}{ll}0 & 0\end{array}$ & $1-$ & -10 & 0 & 0 & 00 & -1 & & 0 & $\begin{array}{ll}0 & 0\end{array}$ & 00 & & & & & \\
\hline$[2, e-2]$ & $\begin{array}{ll}0 & 0\end{array}$ & -1 & $\begin{array}{ll}0 & 0\end{array}$ & $0-$ & -1 & $0 \quad 0$ & 1 & & & & 00 & 01 & & & & \\
\hline$[e-1, e-2$, & $\begin{array}{ll}0 & 0\end{array}$ & $0-$ & -10 & 0 & 1 & $\begin{array}{ll}0 & 0\end{array}$ & 0 & 1 & & $0-1$ & 00 & -1 & & & & \\
\hline$[e-1, e$ & $\begin{array}{ll}0 & 0\end{array}$ & 0 & $0-1$ & & 0 & $\begin{array}{ll}0 & 0\end{array}$ & 0 & 0 & & $\begin{array}{ll}0 & 0\end{array}$ & $\begin{array}{ll}0 & 0\end{array}$ & 0 & & & & \\
\hline & $\begin{array}{ll}0 & 0\end{array}$ & 0 & $\begin{array}{ll}0 & 0\end{array}$ & 0 & & & 0 & 0 & & & 10 & 01 & & & & \\
\hline & & & & & & & & & & & & & & & & \\
\hline
\end{tabular}

B.2. $\mu=[e, e, 2], \quad \mu^{\diamond^{\prime}}=[e-1,1]$.

\begin{tabular}{|c|c|c|c|c|c|c|}
\hline & $\begin{array}{lll} & F \\
0 & 0 & 0 \\
0 & 0 & N\end{array}$ & $\begin{array}{l}\bar{N} \\
-i \\
1 \\
0 \\
0 \\
\dot{0}\end{array}$ & 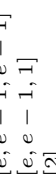 & 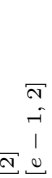 & 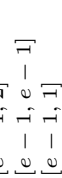 & 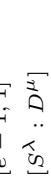 \\
\hline $\begin{array}{l}{[e, e, 2]} \\
{[e, e, 1]} \\
{[2, e]} \\
{[e, e-1,2]} \\
{[e, e-1, e-1]} \\
{[e, e-1,1]} \\
{[2]} \\
{[e-1,2]} \\
{[e-1, e-1]} \\
{[e-1,1]}\end{array}$ & $\begin{array}{lll} & \cdot & \cdot \\
1 & \cdot & . \\
1 & 0 & \\
-1 & 0 & 1 \\
0 & 0 & 1 \\
0 & 1 & 0 \\
1 & 0 & 1 \\
-1 & 0 & 0 \\
0 & 0 & 0 \\
0 & 1 & 0\end{array}$ & $\begin{array}{c} \\
\\
\\
1 \\
-1 \\
0 \\
1 \\
0 \\
0\end{array}$ & 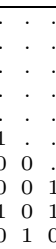 & $\begin{array}{ll}\cdot & . \\
& . \\
. & . \\
. & . \\
1 & . \\
1 & 1 \\
0 & -1\end{array}$ & 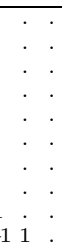 & $\begin{array}{l}1 \\
1 \\
1 \\
0 \\
1 \\
1 \\
1 \\
0 \\
1 \\
1\end{array}$ \\
\hline
\end{tabular}

\section{ACKNOWLEDGEMENT}

An earlier version of this paper was very cumbersome. The present streamlined version incorporates some ideas from the author's forthcoming paper with Kai Meng Tan [5], which was written while the author was visiting the National University of Singapore. The author is very grateful for that institution's hospitality. The author also wishes to thank Kai Meng Tan for pointing out the easy proof of Lemma 3.6.

\section{REFERENCES}

[1] J. Brundan \& A. S. Kleshchev, 'Representation theory of the symmetric groups and their double covers', Groups, combinatorics 8 geometry (Durham, 2001), 31-53, World Sci. Publishing, River Edge, NJ, 2003. MR1994959 (2004i:20016)

[2] J. Chuang \& K. M. Tan, Some canonical basis vectors in the basic $U_{q}\left(\widehat{\mathfrak{s l}}_{n}\right)$-module, J. Algebra 248 (2002), 765-79. MR1882121 (2002k:20011)

[3] R. Dipper \& G. D. James, Representations of Hecke algebras of general linear groups, Proc. London Math. Soc. (3) 52 (1986), 20-52. MR812444 (88b:20065) 
[4] M. Fayers, Weight two blocks of Iwahori-Hecke algebras in characteristic two, Math. Proc. Cambridge Philos. Soc. 139 (2005), 385-397. MR2177166 (2006k:20010)

[5] M. Fayers \& K. M. Tan, Adjustment matrices for weight three blocks of Iwahori-Hecke algebras, J. Algebra 306 (2006), 76-103.

[6] G. D. James, The representation theory of the symmetric groups, Lecture notes in mathematics 682, Springer-Verlag, New York/Berlin, 1978. MR513828 (80g:20019)

[7] G. D. James, The decomposition matrices of $\mathrm{GL}_{n}(q)$ for $n \leq 10$, Proc. London Math. Soc. (3) 60 (1990), 225-265. MR1031453 (91c:20024)

[8] G. D. James, S. Lyle \& A. Mathas, Rouquier blocks, Math. Z. 252 (2006), 511-531. MR2207757 (2006m:20007)

[9] G. D. James \& A. Mathas, A q-analogue of the Jantzen-Schaper theorem, Proc. London Math. Soc. (3) 74 (1997), 241-274. MR1425323 (97j:20013)

[10] G. D. James \& A. Mathas, Equating decomposition numbers for different primes, J. Algebra 258 (2002), 599-614. MR1943936 (2003i:20006)

[11] A. Lascoux, B. Leclerc \& J.-Y. Thibon, Hecke algebras at roots of unity and crystal bases of quantum affine algebras, Comm. Math. Phys. 181 (1996), 205-263. MR1410572 (97k:17019)

[12] B. Leclerc \& H. Miyachi, Some closed formulas for canonical bases of Fock spaces, Represent. Theory 6 (2002), 290-312. MR1927956 (2004a:17022)

[13] S. Martin \& L. Russell, Defect 3 blocks of symmetric group algebras, J. Algebra 213 (1999), 304-339. MR1674687 (99k:20028)

[14] A. Mathas, Iwahori-Hecke algebras and Schur algebras of the symmetric group, University lecture series 15, American Mathematical Society, Providence, RI, 1999. MR1711316 (2001g:20006)

[15] G. Mullineux, Bijections on p-regular partitions and $p$-modular irreducibles of the symmetric groups, J. London Math. Soc. (2) 20 (1979), 60-66. MR545202 (80j:20016)

[16] M. J. Richards, Some decomposition numbers for Hecke algebras of general linear groups, Math. Proc. Cambridge Philos. Soc. 119 (1996), 383-402. MR1357053 (97d:20009)

[17] J. C. Scopes, Cartan matrices and Morita equivalence for blocks of the symmetric groups, $J$. Algebra 142 (1991), 441-455. MR1127075 (92h:20023)

School of Mathematical Sciences, Queen Mary, University of London, Mile End RoAd, London E1 4NS, United Kingdom 